\title{
Fundamental groups of topological stacks with the slice property
}

\author{
BEHRANG NOOHI
}

\begin{abstract}
The main result of the paper is a formula for the fundamental group of the coarse moduli space of a topological stack. As an application, we find simple formulas for the fundamental group of the coarse quotient of a group action on a topological space in terms of the fixed point data. In particular, we recover, and vastly generalize, results of Armstrong [1; 2], Bass [3], Higgins and Taylor [7] and Rhodes [11].
\end{abstract}

54H15, 22A22, 22F05; 57S30, 57S20, 57S10, 57S05

\section{Introduction}

The purpose of this paper is to prove a basic formula for the fundamental group of the coarse moduli stack of a topological stack (Theorem 8.3). This result has consequences in classical algebraic topology which seem, surprisingly, to be new. (Some special cases have appeared previously in Armstrong [1;2], Higgins and Taylor [7] and Rhodes [11]. Also see Section 9.10 of Brown [5] which treats a related result of Brown and Higgins.) They give rise to simple formulas for the fundamental group of the coarse quotient space of a group action on a topological space in terms of the fixed point data of the action; see Theorem 9.1, Theorem 10.4 and Remark 10.7 - Corollary 10.5 should also be of interest.

For the above results to hold, one needs a technical hypothesis which is called the slice property (Definitions 5.1 and 5.4). As the terminology suggests, this notion is modeled on the slice property of compact Lie group actions. Stacks which satisfy the slice property include quotient stacks of proper Lie groupoids and stack that are (locally) quotients of Cartan $G$-spaces (see Example 2 in Section 5.1), where $G$ is an arbitrary Lie group. The latter case includes: 1) Deligne-Mumford topological stacks, hence, all orbifolds; 2) quotient stacks of compact Lie group actions on completely regular spaces; 3) quotient stacks of proper Lie group actions on locally compact spaces.

Roughly speaking, the idea behind the above theorems is that, in the presence of the slice property, the fundamental group of the coarse moduli space (or the coarse quotient of a group action) is obtained by simply killing the loops which "trivially" die as we 
pass to the coarse space. For example, we know that under the moduli map $\mathcal{X} \rightarrow \mathcal{X}_{\text {mod }}$ the ghost loops (or inertial loops) in $\pi_{1}(\mathcal{X})$ die. Theorem 8.3 says, roughly, that $\pi_{1}\left(\mathcal{X}_{\bmod }\right)$ is obtained precisely by killing all the ghost loops in $\pi_{1}(\mathcal{X})$.

Theorem 8.3 can be applied in a variety of situations, eg, when $\mathcal{X}$ is a complex-ofgroups, an orbifold, the leaf stack of a foliation, and so on. In the case where $\mathcal{X}$ is a graph-of-groups, this recovers a result of Bass [3, Example 2.14].

Our strategy in proving these results is to make a systematic use of the covering theory of topological stacks, which we developed in [9, Section 18]. This approach has the advantage that it is neat and it minimizes the use of path chasing arguments. The major players in the game are the maps $\omega_{x}: I_{x} \rightarrow \pi_{1}(\mathcal{X}, x)$ introduced in [9, Section 17] which realize elements of the inertia groups $I_{x}$ as ghost loops in $\mathcal{X}$.

Organization of the paper To be able to make use of the formalism of Galois categories, in the first part of Section 2 we go over prodiscrete topologies on groups and prodiscrete completions. (A prodiscrete topology on a group is a topology which admits a basis at the identity consisting of subgroups (not necessarily normal); pro-Ctopologies of Ribes [12] are examples of these.) This is presumably standard material. In the second part of Section 2, we remind the reader of Grothendieck's theory of Galois categories.

Main examples of Galois categories arise from covering stacks, and they give rise to prodiscrete groups. This is discussed in Section 3. In Section 4 we introduce the Galois categories that interest us in this paper. Understanding these Galois categories is the key in proving our results about fundamental groups of stacks.

Up to this point in the paper, everything is quite formal and we do not make any assumptions on our stacks (other than being connected and locally path-connected). In Section 5, we introduce topological stacks with the slice property (Definition 5.1 and Definition 5.4). In Section 6, we look at a certain class of stacks with the slice property which satisfy a certain locally path-connectedness condition. We call them strongly locally path-connected stacks. These are stacks whose covering theory is as well-behaved as it can be.

The interrelationship between the various Galois categories introduced in Section 4 is discussed in Section 7. The slice property will play an important role in relating these Galois theories.

In Section 8, we translate the results of Section 4 in terms of fundamental groups and obtain our first main result, Theorem 8.3. 
In Section 9 and Section 10, we apply Theorem 8.3 to the quotient stack $[X / G]$ of a group action and derive our next main results, Theorem 9.1 and Theorem 10.4.

In Section 11, we compare our results with those of Armstrong [2].

\section{Some abstract Galois theory}

In this section, we review Grothendieck's theory of Galois categories, slightly modified so we do not need the finiteness assumptions of SGA1 [6].

Throughout the paper, all group actions are on the left and are continuous.

\subsection{Prodiscrete completions}

To set up a Galois theory that is general enough for our purposes, we need to extend the theory of profinite groups so that it applies to topologies generated by families of subgroups that are not necessarily of finite index. Lack of compactness causes a bit of technical difficulty, but it does not effect the outcome, at least as far as our applications are concerned. The material in this subsection should be standard.

Definition 2.1 Let $G$ be a topological group. We say that the topology of $G$ is prodiscrete if its open subgroups form a fundamental system of neighborhoods at the identity.

Let $G$ be a group, and let $\mathcal{H}$ be a family of subgroups of $G$ satisfying the following axioms:

(Top1) If $H_{1}, H_{2} \in \mathcal{H}$, then $H_{1} \cap H_{2} \in \mathcal{H}$.

(Top2) For any $H \in \mathcal{H}$ and any $g \in G, g \mathrm{Hg}^{-1}$ is in $\mathcal{H}$.

(Top3) If $H \in \mathcal{H}$ and $H \subseteq H^{\prime}$, then $H^{\prime} \in \mathcal{H}$.

In this case, there is a prodiscrete topology on $G$ with the property that a subgroup $H \subseteq G$ is open if and only if $H \in \mathcal{H}$. We sometime refer to this topology as the $\mathcal{H}$-topology on $G$.

The $\mathcal{H}$-topology is Hausdorff if and only if $\bigcap_{\mathcal{H}} H=\{1\}$. In this case, the topology is totally disconnected. It is compact if and only if every $H \in \mathcal{H}$ has finite index in $G$. In this case, there exists a fundamental system of neighborhoods at the identity consisting of normal subgroups.

Algebraic 83 Geometric Topology, Volume 8 (2008) 
Example 2.2 (1) For an arbitrary topological group $G$, there is a natural choice of a prodiscrete topology on $G$, namely, the one where $\mathcal{H}$ is the collection of all open-closed subgroups of $G$. We call this the canonical prodiscrete topology on $G$. There is another natural topology on $G$ generated by normal open-closed subgroups. These two topologies are in general not the same.

(2) A given discrete group $G$ can be endowed with several prodiscrete topologies. For instance, the profinite, prosolvable, pronilpotent and pro- $p$ topologies. The discrete topology is also prodiscrete. In general, for any formation $\mathrm{C}$ of groups (that is, a collection of groups closed under taking quotients and finite subdirect products), one can consider the pro-C topology on a group $G$. In the pro-C topology, $H \subseteq G$ is open if $H$ is normal and $G / H$ is in C.

All these topologies have a basis consisting of normal subgroups.

(3) Given a family $\mathcal{H}$ of subgroups of a group $G$, there is a smallest prodiscrete topology on $G$ in which every $H \in \mathcal{H}$ is open. We call this the topology generated by $\mathcal{H}$. Open subgroups in this topology are subgroups $K \subseteq G$ which contain some finite intersection of conjugates of groups in $\mathcal{H}$. When $\mathcal{H}$ consists of a single normal subgroup $N$, then the open subgroups of the topology generated by $N$ are exactly the subgroups of $G$ which contain $N$.

Let $G$ be an arbitrary topological group. We define $G$-Set to be the category of all continuous discrete $G$-sets. There is a forgetful functor $F_{G}$ : $G-$ Set $\rightarrow$ Set.

Convention Throughout the paper all $G$-sets are assumed to be continuous and discrete.

Definition 2.3 Let $G$ be an arbitrary topological group. We define the prodiscrete completion of $G$ to be $G^{\wedge}:=\operatorname{Aut}\left(F_{G}\right)$, where $\operatorname{Aut}\left(F_{G}\right)$ is the group of self-transformations of the forgetful functor $F_{G}$ : $G$-Set $\rightarrow$ Set. When $G$ is given by an $\mathcal{H}$-topology, we will also use the notation $G_{\mathcal{H}}^{\wedge}$.

Remark 2.4 The prodiscrete completion of a topological group $G$ is the same as the prodiscrete completion of $G$ endowed with its canonical prodiscrete topology (Example 2.2(1)). Therefore, it is natural to restrict to prodiscrete topological groups when talking about prodiscrete completions.

There is a natural homomorphism $\iota_{G}: G \rightarrow G^{\wedge}$ which is characterized by the property that, for every $X \in G$-Set, the actions of $G$ and $G^{\wedge}$ on $X$ are compatible with each other via $\iota_{G}$. The map $\iota_{G}$ is injective if and only if $G$ is Hausdorff. 
We endow $G^{\wedge}$ with a prodiscrete topology in which the open subgroups are those subgroups of $\operatorname{Aut}\left(F_{G}\right)=G^{\wedge}$ which are of the form $U_{X, x}$, for $X \in G-$ Set and $x \in X$. Here, $U_{X, x}$ stands for the group of all elements in $\operatorname{Aut}\left(F_{G}\right)$ whose action on $X$ leaves $x$ fixed.

Proposition 2.5 The subgroups $U_{X, x} \subseteq G^{\wedge}$, where $X \in G$-Set and $x \in X$, are exactly the open subgroups of a prodiscrete topology on $G^{\wedge}$.

Proof We have to check the axioms (Top1), (Top2) and (Top3).

(Top1) This follows from the equality $U_{X, x} \cap U_{Y, y}=U_{X \times Y,(x, y)}$. The proof of this equality is easy, but there is a tiny subtlety. The point is that, there are, a priori, two actions of $G^{\wedge}$ on $X \times Y$. One is the componentwise action on the product. The other is obtained by considering $X \times Y$ as an object in $G$-Set and then taking the induced action of $G^{\wedge}=\operatorname{Aut}\left(F_{G}\right)$ on it; see Definition 2.3. These two actions are, however, identical, as can be seen by considering the two ( $G$-equivariant) projection maps $\mathrm{pr}_{1}: X \times Y \rightarrow X$ and $\mathrm{pr}_{2}: X \times Y \rightarrow X$ and using the fact that every $\gamma \in G^{\wedge}$, being a transformation of functors, should respect $\mathrm{pr}_{1}$ and $\mathrm{pr}_{2}$.

(Top2) For every $\gamma \in G^{\wedge}$ and every $U_{X, x}$, we have $\gamma U_{X, x} \gamma^{-1}=U_{X, \gamma x}$.

(Top3) Note that in the definition of $U_{X, x}$, we may assume that the action of $G$ on $X$ is transitive (because $U_{X, x}=U_{G \cdot x, x}$ ). This implies that the action of $G^{\wedge}$ on $X$ is also transitive. Let $U \subseteq G^{\wedge}$ be a subgroup that contains $U_{X, x}$. We have to construct a $G$-set $Y$ and a point $y \in Y$ such that $U$ is exactly the stabilizer of $y$ in $G^{\wedge}$.

Let $A:=U \cdot x \subseteq X$ be the orbit of $x$ under the action of $U$. It is easy to see that, for every $\gamma, \gamma^{\prime} \in G^{\wedge}$, either $\gamma \cdot A=\gamma^{\prime} \cdot A$ or $\gamma \cdot A \cap \gamma^{\prime} \cdot A=\varnothing$; the equality happens exactly when $\gamma$ and $\gamma^{\prime}$ are in the same left coset of $U$ in $G^{\wedge}$. Since the action of $G^{\wedge}$ on $X$ is transitive, this partitions $X$ into translates of $A$. Let $Y$ be the set of equivalence classes. (In other words, $Y$ is just the set $G^{\wedge} / U$.) We have an induced action of $G^{\wedge}$ on $Y$, hence also one of $G$ on $Y$. This way, $Y$ becomes a $G$-set. Under the action of $G^{\wedge}$, the stabilizer group of the class of $A$ in $Y$ is exactly $U$. This completes the proof.

There is a tiny subtlety in the above argument that needs some explanation. Note that $Y$, viewed as an object of $G$-Set inherits an action of $\operatorname{Aut}\left(F_{G}\right)=G^{\wedge}$ which, a priori, may be different from the original action of $G^{\wedge}$ on it. However, these two action are actually the same. This can be seen by looking at the projection map $p: X \rightarrow Y$, viewed as a morphism in $G$-Set, and using the fact elements of $G^{\wedge}=\operatorname{Aut}\left(F_{G}\right)$ are transformations of functors (hence respecting $p$ ). 
The following lemma is immediate.

Lemma 2.6 The map $U \mapsto \iota_{G}^{-1}(U)$ induces a bijection between open subgroups $U$ of $G^{\wedge}$ and open subgroups of $G$. This bijection sends normal subgroups to normal subgroups. For every open subgroup $U \subseteq G^{\wedge}$, we have a bijection $G / \iota_{G}^{-1}(U) \stackrel{\sim}{\longrightarrow}$ $G^{\wedge} / U$. In particular, the map $\iota_{G}: G \rightarrow G^{\wedge}$ is continuous. Indeed, the topology on $G^{\wedge}$ is the finest topology that makes $\iota_{G}$ continuous. Furthermore, the natural functor $\iota_{G}^{*}: G^{\wedge}-$ Set $\rightarrow G-$ Set is an equivalence of categories. Finally, $\iota_{G}^{*}$ respects the forgetful functors $F_{G}: G$-Set $\rightarrow$ Set and $F_{G^{\wedge}}: G^{\wedge}-$ Set $\rightarrow$ Set. That is, the diagram

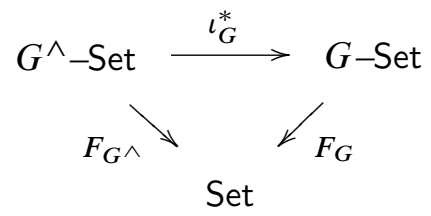

is commutative.

The lemma implies that any continuous action of $G$ on a set $X$ extends uniquely to a continuous action of $G^{\wedge}$ on $X$, and every $G$-equivariant map $X \rightarrow Y$ is automatically $G^{\wedge}$-equivariant. The group $G^{\wedge}$ is universal among the topological groups that have the same category of $G$-sets as $G$.

Definition 2.7 We say that a prodiscrete group $G$ is complete if $\iota_{G}: G \rightarrow G^{\wedge}$ is an isomorphism.

Proposition 2.8 For every prodiscrete group $G$ the prodiscrete completion $G^{\wedge}$ is complete.

Proof By Lemma 2.6, the category $G^{\wedge}$-Set is equivalent to $G$-Set via an equivalence that respects the forgetful functors. This equivalence induces a natural isomorphism between the groups of self-transformations of the two forgetful functors.

Proposition 2.9 Every discrete group is complete.

Proof Pick a self-transformation $\gamma \in \operatorname{Aut}\left(F_{G}\right)$ of the forgetful functor $F_{G}: G$-Set $\rightarrow$ Set. We have to show that there exists $g \in G$ such that for every $G-$ Set $X$ the action of $\gamma$ on $X$ is the same as the action of $g$. It is enough to assume that $X=G / H$, for some subgroup $H$ of $G$. (This is because if the actions of $g$ and $\gamma$ coincide for every transitive $G$-set, then they coincide for every $G$-set.) In fact, since $G \rightarrow G / H$ is surjective, it is enough to assume that $X=G$ with the left multiplication action. 
Let $G_{\text {triv }}$ be the set $G$ with the trivial $G$-action. Since the action of $\gamma$ on a point is trivial, its action on $F_{G}\left(G_{\text {triv }}\right)$ is also trivial. By considering the action of $\gamma$ on the diagram of $G$-sets

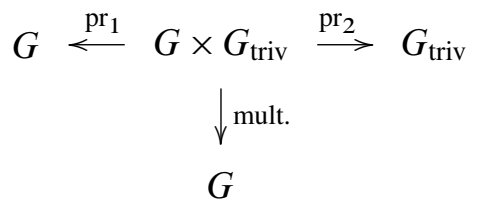

it follows that the action of $\gamma$ respects right multiplication by every element of $G$. Therefore, $\gamma$ must be equal to left multiplication by the element $g:=\gamma(1) \in G$.

Example 2.10 (1) Let $G$ be a group, and let $N \subseteq G$ be a normal subgroup. Endow $G$ with the prodiscrete topology in which open subgroups are exactly the ones containing $N$. Then $G^{\wedge} \cong G / N$.

(2) Let $G$ be a finite group. Then every prodiscrete topology on $G$ is of the form above. To see this, take $N$ to be the intersection of all open subgroups of $G$.

Given a continuous group homomorphism $f: G \rightarrow H$, we have an induced continuous homomorphism $f^{\wedge}: G^{\wedge} \rightarrow H^{\wedge}$ making the following square commute:

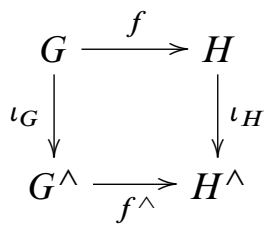

Corollary 2.11 Let $f: G \rightarrow H$ be a continuous homomorphism, where $G$ is a prodiscrete group and $H$ is a discrete group. Then there is a unique extension $f^{\wedge}: G^{\wedge} \rightarrow H$.

Proof By functoriality of $\iota$ we have a natural homomorphism $f^{\wedge}: G^{\wedge} \rightarrow H^{\wedge}$. The assertion follows from Proposition 2.9.

Of course, the above statement is true for any complete prodiscrete group $H$.

Lemma 2.12 Let $G$ be a prodiscrete group, and let $G^{\prime}$ be another prodiscrete group whose underlying group is the same as $G$ but whose topology is weaker. Note that, by Lemma 2.6, this induces a weaker topology on $G^{\wedge}$, which we denote by $G^{\prime \prime}$. Then the natural (continuous) homomorphism $G^{\prime} \rightarrow G^{\prime \prime}$ induces an isomorphism $G^{\prime \wedge} \stackrel{\sim}{\longrightarrow} G^{\prime \prime \wedge}$. (Note that when $G=G^{\prime}$ we recover Proposition 2.8.) 
Proof The categories $G^{\prime}$-Set and $G^{\prime \prime}-$ Set are naturally subcategories of $G$-Set and $G^{\wedge}-$ Set, respectively, and the restriction of the equivalence $\iota_{G}^{*}: G^{\wedge}-$ Set $\rightarrow G-$ Set to $G^{\wedge}$-Set induces an equivalence $G^{\prime \prime \wedge}-$ Set $\rightarrow G^{\prime}-$ Set (respecting the forgetful functors). This gives us the desired isomorphism $G^{\prime \wedge} \stackrel{\sim}{\longrightarrow} G^{\prime \prime \wedge}$.

Remark 2.13 It seems that it is not true in general that the image of $\iota_{G}: G \rightarrow G^{\wedge}$ is dense, unless we assume that $G$ has a basis consisting of normal open subgroups (see Proposition 2.14). All we can say in general is that there is no proper open subgroup of $G^{\wedge}$ containing $\iota_{G}(G)$.

Proposition 2.14 Assume that $G$ is a prodiscrete group that has a basis $\mathcal{N}=\left\{N_{i}\right\}_{i \in I}$ (around identity) consisting of normal subgroups. Then there is a natural isomorphism

$$
G^{\wedge} \cong \underset{\overleftarrow{\mathcal{N}}}{\lim } G / N_{i}
$$

In other words, in this case our notion of completion coincides with the classical one.

Proof Denote the right hand side by $\widetilde{G}$. It is easy to check that the map $G \rightarrow \widetilde{G}$ induces an equivalence of categories $\widetilde{G}-$ Set $\rightarrow G$-Set (respecting the forgetful functors $F_{G}$ and $F_{\widetilde{G}}$ ). So, it is enough to show that $\widetilde{G}$ is complete, ie, $\iota_{\widetilde{G}}: \widetilde{G} \rightarrow$ Aut $F_{\widetilde{G}}$ is an isomorphism.

This map is injective, since $\widetilde{G}$ is Hausdorff. To prove the surjectivity, let $\alpha \in$ Aut $F_{\widetilde{G}}$. Fix an $i \in I$ and consider the $\widetilde{G}$-set $G / N_{i}$. The action of $\alpha$ on $G / N_{i}$ sends $1 \in G / N_{i}$ to some $g_{i} \in G / N_{i}$. For any $h \in G / N_{i}$, multiplication on the right by $h$ induces a map of $\widetilde{G}$-sets $G / N_{i} \rightarrow G / N_{i}$. Therefore, since the action of $\alpha$ is functorial, its effect on $G / N_{i}$ sends $h=1 h$ to $g_{i} h$. That is, $\alpha$ acts by multiplication on the left by $g_{i}$. Again, by the functoriality of the action of $\alpha$, the various $g_{i}$ are compatible, that is, they come from an element

$$
g \in{\underset{\lim }{\mathscr{N}}} G / N_{i}=\widetilde{G}
$$

This proves the surjectivity.

\subsection{Review of Grothendieck's Galois theory}

We review (a slightly modified version of) Grothendieck's theory of Galois categories [6]. The difference here is that we want to apply the theory to the cases where the covering maps are not necessarily finite, so we will remove certain finiteness assumptions. 
Definition 2.15 (see SGA [6] for more details) By a Galois category we mean a pair $(\mathrm{C}, F)$, where $\mathrm{C}$ is a category and $F: \mathrm{C} \rightarrow$ Set is a functor, satisfying the following axioms: ${ }^{1}$

(G1) The category $\mathrm{C}$ has finite projective limits (ie, $\mathrm{C}$ has a final object and fiber products exist).

(G2) Direct sums (not necessarily finite) exist. In particular, an initial object exists. Also, quotient of an object under an equivalence relation exists. In particular, quotients under (faithful) group actions exist.

(G3) Let $u: X \rightarrow Y$ be a morphism in C. Then $u$ factorizes as $X \stackrel{u^{\prime}}{\longrightarrow} Y^{\prime} \stackrel{u^{\prime \prime}}{\longrightarrow} Y$, with $u^{\prime}$ a strict epimorphism and $u^{\prime \prime}$ a monomorphism that is an isomorphism onto a direct summand of $Y$.

(G4) The functor $F$ is left exact. That is, it commutes with fiber products and takes the final object to the final object.

(G5) The functor $F$ commutes with direct sums, takes strict epimorphisms to epimorphisms and commutes with taking quotients (as in (G2)).

(G6) The functor $F$ is conservative. That is, if $u: X \rightarrow Y$ is a morphism in C such that $F(u)$ is an isomorphism, then $u$ is an isomorphism.

The functor $F$ is called the fundamental functor. A functor between Galois categories is called a Galois functor if it respects the fundamental functors. An object in a Galois category is called connected if it can not be written as a direct sum of two objects. An example of a Galois category is the category of continuous $G$-sets, where $G$ is an arbitrary topological group. The fundamental functor in this case is the forgetful functor. The main theorem of Grothendieck's Galois theory is that this is basically the only example.

Theorem 2.16 Let $(\mathrm{C}, F)$ be a Galois category. Let $\pi_{1}^{\prime}(\mathrm{C}, F):=$ Aut $F$ be the (complete prodiscrete) group of automorphisms of $F$. Then there is a natural equivalence of Galois categories $\mathrm{C} \cong \pi_{1}^{\prime}(\mathrm{C}, F)-$ Set. $^{2}$

The above equivalence is functorial with respect to functors between Galois categories. In other words, the category of Galois categories and Galois functors between them is equivalent to the category of complete prodiscrete groups and continuous group homomorphisms.

\footnotetext{
${ }^{1}$ The axioms are numbered in this way to be compatible with [6].

${ }^{2}$ The reason for using the notation $\pi_{1}^{\prime}$ becomes clear when we consider the Galois category associated to a topological space $X$ (Section 4.1), in which case $\pi_{1}^{\prime}$ and $\pi_{1}$ will not in general be the same unless we assume that $X$ is semilocally 1 -connected.
} 
The proof of the above theorem is just a slight modification of the proof given in [6] and we omit it.

An object $X$ in a Galois category $(C, F)$ is called Galois if $X /$ Aut $X=*$. This means that the group of automorphisms of $X$ (which we think of as the Galois group of $X$ ) is as big as it can be. For example, in $G-$ Set every $G / N$, where $N$ is an open normal subgroup of $G$, is a Galois object (and every connected Galois object is of this form). In general, connected Galois objects are in bijection with open normal subgroups of $\pi_{1}^{\prime}(\mathrm{C}, F)$.

Note that, in Theorem 2.16 we did not require the existence of Galois objects in C, although it will be the case in most examples. In fact, in most situations, one can find a cofinal family of connected Galois objects (ie, every connected object is dominated by a connected Galois object). This is equivalent to saying that $\pi_{1}^{\prime}(\mathrm{C}, F)$ has a basis at the identity consisting of normal subgroups. In this situation, Proposition 2.14 implies that $\pi_{1}^{\prime}(C, F)$ can be computed as the opposite of the inverse limit of the Galois groups of the Galois objects. (The reason we have to take the opposite is that the group of automorphisms of the object $G / N \in G-$ Set is $(G / N)^{\mathrm{op}}$.) Let us summarize this in the following proposition.

Proposition 2.17 Let (C, F) be a Galois category, and let $\left\{X_{i}\right\}_{i \in I}$ be a cofinal family of connected Galois objects (ie, every connected object is dominated by some $X_{i}$ ). Then we have a natural isomorphism

$$
\pi_{1}^{\prime}(\mathrm{C}, F) \cong \lim _{I}\left(\text { Aut } X_{i}\right)^{\mathrm{op}}
$$

\section{Galois theory of covering stacks}

The Galois theory of covering stacks of a topological stack $\mathcal{X}$ is closely related to the group theory of its fundamental group. But, these two theories can diverge unless we assume that $\mathcal{X}$ behaves nicely locally: the mouthful "semilocally 1-connected" condition. This property, unfortunately, may not be preserved under certain natural constructions that one makes with topological stacks (say, base extension, or passing to the coarse moduli space). To avoid this nuisance we begin by developing a theory of fundamental groups which is more in tune with Galois theory of covering stacks. We then explain how it relates to the usual theory of fundamental groups. 


\subsection{Review of topological stacks}

We recall a few definitions from [9]. We follow the notational convention of [9] by using calligraphic symbols $\mathcal{X}, \mathcal{Y}, \mathcal{Z}, \ldots$ for stacks and script symbols $X, Y, Z, \ldots$ for spaces.

Throughout the paper, a stack means a stack over the site Top of topological spaces; here, Top is endowed with its standard open-cover topology. A stack $\mathcal{X}$ is called topological if it is equivalent to the quotient stack of a topological groupoid $\left[X_{1} \rightrightarrows X_{0}\right.$ ] whose source maps is a local Serre fibration in the sense of [9, Section 13.1]. An atlas ${ }^{3}$ for a topological stack $\mathcal{X}$ is an epimorphism $p: X \rightarrow \mathcal{X}$ from a topological space $X$. Given such an atlas, one finds a groupoid presentation for $\mathcal{X}$ by taking $X_{1}=X \times_{\mathcal{X}} X$ and $X_{0}=X$.

Every topological space $X$ can be thought of as a topological stack, namely, the topological stack associated to the trivial groupoid $[X \rightrightarrows X]$. This gives a fully faithful embedding of the category of topological spaces and continuous maps to the category of topological stacks. Also, for every topological group $G$, every continuous $G$-space gives rise to a topological stack $[X / G]$. Among other examples of topological stacks we mention orbifolds, the underlying topological stacks of Artin stacks, complexes-ofgroups and leaf stacks of foliations.

The basic notions of algebraic topology (eg, homotopy, homotopy groups, generalized homology/cohomology, fibrations, mapping spaces, loop spaces, etc.) generalize to topological stacks.

By a point $x$ in a stack $\mathcal{X}$ we mean a morphism $x: * \rightarrow \mathcal{X}$ of stacks. The inertia group of a point $x$ is the group of self-transformations of the above map. It is naturally a topological group when $\mathcal{X}$ is topological, and is denoted by $I_{x}$.

To a topological stack $\mathcal{X}$ one associates a topological space $\mathcal{X}_{\text {mod }}$, called the coarse moduli space of $\mathcal{X}$. There is a natural morphism $\pi: \mathcal{X} \rightarrow \mathcal{X}_{\text {mod }}$ that is universal among morphisms from $\mathcal{X}$ to topological spaces. The map $\pi$ induces a natural bijection between the set of 2-isomorphism classes of points of $\mathcal{X}$ and the set of points of $\mathcal{X}_{\text {mod }}$. When $\mathcal{X}=\left[X_{0} / X_{1}\right]$ for a topological groupoid $\left[X_{1} \rightrightarrows X_{0}\right], \mathcal{X}_{\text {mod }}$ is naturally homeomorphic to the coarse quotient space $X_{0} / X_{1}$. In particular, when $\mathcal{X}=[X / G]$ is the quotient stack of a group action, the coarse moduli space $\mathcal{X}_{\text {mod }}$ is homeomorphic to the coarse quotient space $X / G$.

\footnotetext{
${ }^{3}$ In [9] we call this a chart. Bad terminology!
} 


\subsection{Review of covering stacks}

We review a few basic facts about covering stacks of topological stacks. More details and proofs can be found in our preprint [9, Section 18].

Definition 3.1 Let $\mathcal{X}$ be a topological stack. We say that $\mathcal{X}$ is connected if it has no proper open-closed substack. We say $\mathcal{X}$ is path-connected, if for every two points $x$ and $y$ in $\mathcal{X}$, there is a path from $x$ to $y$.

Definition 3.2 Let $\mathcal{X}$ be a topological stack. We say that $\mathcal{X}$ is locally connected (respectively, locally path-connected, semilocally 1-connected), if there is an atlas $X \rightarrow \mathcal{X}$ such that $X$ is so.

These definitions agree with the usual definitions when $\mathcal{X}$ is a topological space. This is because of the following lemma.

Lemma 3.3 Let $f: Y \rightarrow X$ be a continuous map of topological spaces that admits local sections. Assume that $Y$ is locally connected (respectively, locally path-connected, semilocally 1-connected). Then so is $X$.

Definition 3.4 A representable map $\mathcal{Y} \rightarrow \mathcal{X}$ of topological stacks is called a covering map if for every topological space $W$ and every map $W \rightarrow \mathcal{X}$, the base extension $W \times \mathcal{X} \mathcal{Y} \rightarrow W$ is a covering map of topological spaces.

Proposition 3.5 Let $f: \mathcal{Y} \rightarrow \mathcal{X}$ be a covering map of topological stacks. Then the diagonal map $\Delta: \mathcal{Y} \rightarrow \mathcal{Y} \times \mathcal{X} \mathcal{Y}$ is an open-closed embedding.

An immediate corollary of this proposition is the following.

Corollary 3.6 Let $f: \mathcal{Y} \rightarrow \mathcal{X}$ be a covering map of topological stacks. Let $p: X \rightarrow \mathcal{X}$ be an atlas for $\mathcal{X}$, and let $q: Y \rightarrow \mathcal{Y}$ be the pullback atlas for $\mathcal{Y}$, where $Y=\mathcal{Y} \times \mathcal{X} X$. (Note that we can also view $Y$ as an atlas for $\mathcal{X}$ via $f \circ q: Y \rightarrow \mathcal{X}$.) Set $R=Y \times \mathcal{Y} Y$ and $R^{\prime}=Y \times \mathcal{X} Y$, and consider the corresponding groupoids $[R \rightrightarrows Y]$ and $\left[R^{\prime} \rightrightarrows Y\right]$ (so $[Y / R] \cong \mathcal{Y}$ and $\left[Y / R^{\prime}\right] \cong \mathcal{X}$ ). Then $[R \rightrightarrows Y]$ is an open-closed subgroupoid of $\left[R^{\prime} \rightrightarrows Y\right]$. 


\subsection{C-complete fundamental groups}

In this subsection we look at the topological incarnations of the notions developed in the previous section. Let $\mathcal{X}$ be a connected locally path-connected topological stack, and let $x$ be a point in $\mathcal{X}$. We will not assume yet that $\mathcal{X}$ is semilocally 1 -connected.

To $(\mathcal{X}, x)$ we can associate various Galois categories $(C, F)$ of covering stacks by requiring the following:

(C1) If $\mathcal{Y}$ is in $\mathrm{C}$ and $\mathcal{Y}^{\prime}$ is another covering stack of $\mathcal{X}$ that is dominated by $\mathcal{Y}$ (ie, there is a surjection $\mathcal{Y} \rightarrow \mathcal{Y}^{\prime}$ relative to $\mathcal{X}$ ), then $\mathcal{Y}^{\prime}$ is in $\mathrm{C}$.

(C2) $\mathrm{C}$ is closed under fiber products.

(C3) C is closed under taking disjoint unions.

(C4) If $\mathcal{Y}$ is in $\mathrm{C}$, then every connected component of $\mathcal{Y}$ is also in $\mathrm{C}$.

The fundamental functor $F$ for such a category is simply the fiber functor $\mathcal{Y} \mapsto \mathcal{Y}_{x}=$ $* \times_{x} \mathcal{Y}$. Axioms $(\mathrm{G} 1)-(\mathrm{G} 6)$ are easy to verify. (Perhaps $(\mathrm{G} 2)$ is a bit nontrivial. It follows from Lemma 3.7 below.)

For a given (connected) covering stack $f: \mathcal{Y} \rightarrow \mathcal{X}$, and for a choice of a basepoint $y$ in $\mathcal{Y}$ lying above $x$ (ie, $y$ is a point in the fiber $\mathcal{Y}_{x}$ of $\mathcal{Y}$ over $x$ ), we have an injection of fundamental groups $\pi_{1}(\mathcal{Y}, y) \rightarrow \pi_{1}(\mathcal{X}, x)$. The image of this injection uniquely determines $(\mathcal{Y}, y)$ up to isomorphism. Changing the base point $y$ will change this subgroup by conjugation.

Let $\mathcal{H}_{\mathrm{C}}$ be the collection of all such subgroups of $\pi_{1}(\mathcal{X}, x)$. It is easily seen that the axioms (Top1)-(Top3) of Section 2.1 are satisfied: (Top1) follows from (C2); (Top2) follows from the discussion of the previous paragraph about changing the base point; (Top3) follows from (C1) and Corollary 3.8 below.

Lemma 3.7 Let $(\mathcal{X}, x)$ be a pointed topological stack, and let $f: \mathcal{Y} \rightarrow \mathcal{X}$ be an arbitrary covering stack of $\mathcal{X}$.

(i) Let $\mathcal{Z}$ be a covering stack of $\mathcal{X}$, and let $\mathcal{Z} \rightarrow \mathcal{Y} \times \mathcal{X} \mathcal{Y}$ be an equivalence relation on $\mathcal{Y}$ (see Axiom (G2)). Then the quotient $\mathcal{Y}^{\prime}$ of this equivalence relation exists and is a covering stack of $\mathcal{X}$.

(ii) Let $F^{\prime}$ be a $\pi_{1}(\mathcal{X}, x)$-set, and let $F_{\mathcal{X}}(\mathcal{Y}) \rightarrow F^{\prime}$ be a surjective $\pi_{1}(\mathcal{X}, x)$ equivariant map, where $F_{x}(\mathcal{Y})$ is the fiber of $f$ over $x$. Then there exists a unique covering stack $\mathcal{Y}^{\prime} \rightarrow \mathcal{X}$ of $\mathcal{X}$ whose fiber is isomorphic to $F^{\prime}$ (as a $\pi_{1}(\mathcal{X}, x)$-set), together with a map of covering stacks $\mathcal{Y} \rightarrow \mathcal{Y}^{\prime}$ realizing $F_{x}(\mathcal{Y}) \rightarrow F^{\prime}$. 
Proof (i) Choose an atlas $X \rightarrow \mathcal{X}$ for $\mathcal{X}$. Let $Y \rightarrow \mathcal{Y}$ and $Z \rightarrow \mathcal{Z}$ be the pullback atlases for $\mathcal{Y}$ and $\mathcal{Z}$. Let $\left[R_{X} \rightrightarrows X\right],\left[R_{Y} \rightrightarrows Y\right]$ and $\left[R_{Z} \rightrightarrows Z\right]$ be the corresponding groupoids. Then $Z \rightarrow Y \times{ }_{X} Y$ and $R_{Z} \rightarrow R_{Y} \times R_{X} R_{Y}$ are equivalence relations. Also, note that the maps $Y \rightarrow X, Z \rightarrow X, R_{Y} \rightarrow R_{X}$ and $R_{Z} \rightarrow R_{X}$ are all covering maps (each being the base extension of either $\mathcal{Y} \rightarrow \mathcal{X}$ or $\mathcal{Z} \rightarrow \mathcal{X}$ ). Set $Y^{\prime}:=Y / Z$ and $R_{Y^{\prime}}:=R_{Y} / R_{Z}$. It is easy to see that we have a natural groupoid structure on $\left[R_{Y^{\prime}} \rightrightarrows Y^{\prime}\right]$. The quotient stack $\mathcal{Y}^{\prime}:=\left[Y^{\prime} / R_{Y^{\prime}}\right]$ is the desired quotient of $\mathcal{Y}$ by $\mathcal{Z}$.

(ii) Note that the statement is true for topological spaces. So, as in the previous part, by choosing an atlas $X \rightarrow \mathcal{X}$ we will reduce the problem to the case of topological spaces. Let $Y, R_{X}$ and $R_{Y}$ be as in the previous part. Let $x_{0} \in X$ be a lift of $x$, and let $x_{1} \in R_{X}$ be the corresponding point in $R_{X}$. Note that the maps $Y \rightarrow X$ and $R_{Y} \rightarrow R_{X}$ are base extensions of $f$, so both are covering maps. Furthermore, the fibers $F_{x_{0}}(Y)$ and $F_{X_{1}}\left(R_{Y}\right)$ are, as sets, in natural bijection with $F_{X}(\mathcal{Y})$. The actions of $\pi_{1}\left(X, x_{0}\right)$ and $\pi_{1}\left(R_{X}, x_{1}\right)$ on these sets are obtained from that of $\pi_{1}(\mathcal{X}, x)$ on $F_{x}(\mathcal{Y})$ via the group homomorphisms $\pi_{1}\left(X, x_{0}\right) \rightarrow \pi_{1}(\mathcal{X}, x)$ and $\pi_{1}\left(R_{X}, x_{1}\right) \rightarrow \pi_{1}(\mathcal{X}, x)$, respectively. We are now reduced to the case of topological spaces, with $\mathcal{X}$ replaced by $X$ and $R_{X}$, respectively. So, we can construct a covering space $Y^{\prime}$ of $X$ and a covering space and $R_{Y^{\prime}}$ of $R_{X}$, together with maps $Y \rightarrow Y^{\prime}$ and $R_{Y} \rightarrow R_{Y^{\prime}}$. It is easy to see that there is a natural groupoid structure on $\left[R_{Y^{\prime}} \rightrightarrows Y^{\prime}\right]$. The quotient stack $\mathcal{Y}^{\prime}:=\left[Y^{\prime} / R_{Y^{\prime}}\right]$ is the desired covering stack of $\mathcal{X}$.

Corollary 3.8 Let $(\mathcal{X}, x)$ be a connected pointed topological stack. Let $f:(\mathcal{Y}, y) \rightarrow$ $(\mathcal{X}, x)$ be a connected covering stack, and let $H \subseteq \pi_{1}(\mathcal{X}, x)$ be the corresponding subgroup. Let $H^{\prime} \subseteq \pi_{1}(\mathcal{X}, x)$ be a subgroup containing $H$. Then there exists a (pointed) covering stack $\mathcal{Y}^{\prime}$ of $\mathcal{X}$ corresponding to $H^{\prime}$.

We summarize our discussion by saying that $\mathcal{H}_{\mathrm{C}}$ is exactly the set of open subgroups of a prodiscrete topology on $\pi_{1}(\mathcal{X}, x)$. The category $C$ is equivalent to the category of continuous $\pi_{1}(\mathcal{X}, x)$-sets. If we denote by $\pi_{1}(\mathcal{X}, x)_{\mathrm{C}}$ the completion of $\pi_{1}(\mathcal{X}, x)$ (Definition 2.3) with respect to this topology, we have proved the following:

Proposition 3.9 The notation being as above, there is an equivalence of Galois categories $(\mathrm{C}, F) \cong \pi_{1}(\mathcal{X}, x)_{\mathrm{C}}-$ Set.

We call $\pi_{1}(\mathcal{X}, x)_{\mathrm{C}}$ the $\mathrm{C}$-complete fundamental group of $(\mathcal{X}, x)$.

\section{Main examples of Galois categories}

We list the main examples of Galois categories that we are interested in. More examples can be produced by noting that, if $\mathrm{C}$ and $\mathrm{C}^{\prime}$ satisfy the above axioms, then so does their 
"intersection" $\mathrm{C} \cap \mathrm{C}^{\prime}$. Here, by $\mathrm{C} \cap \mathrm{C}^{\prime}$ we mean the category of all covering stacks $\mathcal{Y} \rightarrow \mathcal{X}$ that are isomorphic to a covering stack in $\mathrm{C}$ and a covering stack in $\mathrm{C}^{\prime}$.

\subsection{The Galois category Full}

The category Full of all covering stacks of $\mathcal{X}$ satisfies the required axioms. This gives the finest $\mathrm{C}$-topology on $\pi_{1}(\mathcal{X}, x)$, which we call the full topology. The corresponding prodiscrete fundamental group $\pi_{1}(\mathcal{X}, x)_{\text {Full }}$ is denoted by $\pi_{1}^{\prime}(\mathcal{X}, x)$. There is a natural homomorphism $\pi_{1}(\mathcal{X}, x) \rightarrow \pi_{1}^{\prime}(\mathcal{X}, x)$. This map is not in general an isomorphism. (This is due to the fact that the universal cover may not exist; see Example 4.1 below). This homomorphism is, however, an isomorphism if $\mathcal{X}$ is connected, locally pathconnected and semilocally 1 -connected, because in this case the universal cover exists [9, Section 18] and the full topology is discrete.

Example 4.1 (Hawaiian earring) Let $X$ be the subspace of $\mathbb{R}^{2}$ defined as the union of circles of radius $2^{-n}, n \in \mathbb{Z}$, centered at the points $\left(2^{-n}, 0\right)$. This space is not semilocally 1 -connected because every open set containing the origin contains all but finitely many of the circles.

Let $Y=\bigvee_{i \in \mathbb{Z}} S^{1}$ be the wedge sum of countably many circles. This is a semilocally 1connected space. We have $\pi_{1}(Y) \cong \mathbb{F}^{\mathbb{Z}}$, the free group on countably many generators $\left\{a_{i} \mid i \in \mathbb{Z}\right\}$. Consider the prodiscrete topology on $\pi_{1}(Y)$ generated by the subgroups $H_{n}$, where $H_{n}$ is the normal subgroup generated by $\left\{a_{i} \mid i>n\right\}$. Let $\pi_{1}(Y)^{\wedge}$ be the prodiscrete completion.

There is a natural continuous bijection $f: Y \rightarrow X$. This map induces an isomorphism $\pi_{1}(Y)^{\wedge} \rightarrow \pi_{1}^{\prime}(X)$. More explicitly,

$$
\pi_{1}^{\prime}(X) \cong \lim _{n \in \mathbb{Z}} \mathbb{F}^{\mathbb{Z}} / H_{n} \cong \lim _{n \in \mathbb{N}} \mathbb{F}^{\leq n}
$$

where $F^{\leq n}$ is the free group on generators $\left\{a_{i} \mid i \leq n\right\}$ and $F^{\leq n+1} \rightarrow F^{\leq n}$ is the map that sends $a_{n+1}$ to 1 .

\subsection{The Galois category Fin}

Another example is the category Fin of covering stacks of $\mathcal{X}$ each of whose connected components has finite degree over $\mathcal{X}$. The corresponding topology on $\pi_{1}(\mathcal{X}, x)$ consists of open sets of the full topology which have finite index in $\pi_{1}(\mathcal{X}, x)$. This topology has a basis of normal subgroups. Therefore, the corresponding completion can be computed (using Proposition 2.14) as

$$
\pi_{1}(\mathcal{X}, x)_{\mathrm{Fin}}^{\wedge} \cong \underset{\mathcal{N}}{\lim } \pi_{1}(\mathcal{X}, x) / N
$$

Algebraic 83 Geometric Topology, Volume 8 (2008) 
where $\mathcal{N}$ is the set of all open (in the full topology) normal subgroups of finite index of $\pi_{1}(\mathcal{X}, x)$. When $\mathcal{X}$ is semilocally 1 -connected (so full topology is discrete) this coincides with the profinite completion of $\pi_{1}(\mathcal{X}, x)$.

\subsection{The Galois category FPR}

Definition 4.2 Let $f: \mathcal{Y} \rightarrow \mathcal{X}$ be a representable morphism of stacks. Let $y \in \mathcal{Y}$ be a point in $\mathcal{Y}$. We say that $f$ is fixed-point-reflecting (FPR for short) at $y$ if the induced map $I_{y} \rightarrow I_{f(y)}$ (which is a priori injective) is an isomorphism. We say that $f$ is FPR if it is FPR at every point.

The following lemma will be used in Section 7.3.

Lemma 4.3 Let $f: \mathcal{Y} \rightarrow \mathcal{X}$ be a representable morphism of stacks, and let $x \in \mathcal{X}$ be a point. Let $\mathcal{Y}_{x}$ be the fiber of $f$ over $x$. Assume that $\mathcal{Y}_{x}$ is finite. Let $\bar{x} \in \mathcal{X}_{\text {mod }}$ be the image of $x$ in $\mathcal{X}_{\text {mod }}$, and let $\mathcal{Y}_{\text {mod, } x}$ be the fiber of $f_{\text {mod }}: \mathcal{Y}_{\text {mod }} \rightarrow \mathcal{X}_{\text {mod }}$ over $\bar{x}$. Then $\#\left(Y_{\bmod , x}\right) \leq \#\left(\mathcal{Y}_{x}\right)$. The equality holds if and only if $f$ is FPR at every point in the fiber of $\mathcal{Y}$ over $x$.

Proof Easy.

It is easy to check that the category FPR of all FPR covering stacks of $\mathcal{X}$ satisfies the axioms (C1)-(C4) of Section 3.3.

\subsection{The Galois category Free}

Let Free $\subseteq$ FPR be the category of all FPR covering stacks $\mathcal{Y} \rightarrow \mathcal{X}$ such that the induced map $\mathcal{Y}_{\text {mod }} \rightarrow \mathcal{X}_{\text {mod }}$ is again a covering map. Then Free satisfies the axioms. We will only prove this in a special case where $\mathcal{X}$ is strongly locally path-connected (Definition 6.1), in which case we will show that Free = FPR (Proposition 7.7).

We will also see in Proposition 7.8 that, under some mild conditions on $\mathcal{X}$, every finite FPR covering stack is automatically free. That is, FPR $\cap$ Fin $=$ Free $\cap$ Fin .

\section{Topological stacks with the slice property}

In this section, we introduce an important class of topological stacks which behave particularly well locally. We call these topological stacks with the slice property. The slice property is the key in proving the main theorems of the paper. We believe this 
is an important property of topological stacks and expect it to be of interest in other situations as well.

Notation Let $G$ be a topological group acting on a space $X$, and let $x$ be a point in $X$. By a slight abuse of notation, we will denote the stabilizer group of $x$ by $I_{x}$. We will view $I_{X}$ as a topological group.

Definition 5.1 (Palais [10, Section 2.1]) Let $G$ be a topological group acting continuously on a topological space $X$, and let $x$ be a point in $X$. A subset $S$ of $X$ is called a slice at $x$ if it has the following properties:

(S1) The subset $I_{x} S \subseteq X$ is open and there exists a $G$-equivariant map $f: G S \rightarrow$ $G / I_{x}$ whose fiber over the point $I_{x} \in G / I_{x}$ is precisely $S$.

(S2) There exists an open subspace $U \subseteq G / I_{x}$ and a local section $\chi: U \rightarrow G$ such that $(u, s) \mapsto \chi(u) s$ is a homeomorphism of $U \times S$ onto an open neighborhood of $x$ in $X$.

We say that the action has the slice property at $x$ if every open neighborhood of $x$ contains a slice at $x$. We say that a group action has the slice property if it has the slice property at every point.

Remark 5.2 (1) It follows from [10, Proposition 2.1.3] that the natural $G$-equivariant map $G \times \times_{I_{x}} S \rightarrow G S$ is a homeomorphism. Here, $G \times_{I_{x}} S$ is the quotient of $G \times S$ under the action of $I_{x}$ defined by $\alpha \cdot(g, x)=\left(g \alpha^{-1}, \alpha x\right)$.

(2) In the case where $G$ is a Lie group (not necessarily compact), (S2) follows from (S1). This is [10, Proposition 2.1.2].

(3) In the case where $I_{x}$ is compact, existence of a slice at $x$ implies existence of slices that are arbitrarily small. Therefore, when the action of $G$ on $X$ has compact stabilizers, to check whether the action has the slice property it is enough to check that there exists at least one slice at every point.

Lemma 5.3 Let $G$ be a topological group acting on a topological space $X$. Let $x$ be a point in $X$ and $S$ a subset containing $x$. Then $S$ is a slice at $x$ if and only if the map of stacks $\left[S / I_{X}\right] \rightarrow[X / G]$ is an open embedding.

Proof We only prove the "only if" part which is what we need in the rest of the paper. We will show that the map $\left[S / I_{X}\right] \rightarrow[X / G]$ identifies $\left[S / I_{X}\right]$ with the open substack $[G S / G]$ of $[X / G]$.

Consider $G \times S$ endowed with the $G \times I_{x}$ action defined by $(g, \alpha) \cdot(h, x):=$ $\left(g h \alpha^{-1}, \alpha x\right)$. Let $\varphi: G \times I_{x} \rightarrow I_{x}$ and $f: G \times S \rightarrow S$ be the projection maps. 
It is clear that the $\varphi$-equivariant map $f$ induces an equivalence of quotient stacks $\left[(G \times S) /\left(G \times I_{x}\right)\right] \rightarrow\left[S / I_{x}\right]$. So, it is enough to show that the map $[(G \times S) /(G \times$ $\left.\left.I_{x}\right)\right] \rightarrow[G S / G]$ is an equivalence of stacks. This map can be written as a composition $\left[(G \times S) /\left(G \times I_{x}\right)\right] \rightarrow\left[\left(G \times I_{I_{x}} S\right) / G\right] \rightarrow[G S / G]$. The first map is obviously an equivalence of stacks. The second map is an equivalence of stacks by virtue of Remark 5.2(1).

Definition 5.4 We say that a topological stack $\mathcal{X}$ has the slice property, if for every point $x$ in $\mathcal{X}$ and every open substack $\mathcal{U} \subseteq \mathcal{X}$ containing $x$, there is an open substack $\mathcal{V} \subseteq \mathcal{U}$ such that $\mathcal{V} \cong\left[V / I_{x}\right]$, where $V$ is a topological space with an action of $I_{x}$ that has the slice property at $x$. In the case where $I_{x}$ are discrete groups, such stacks are called Deligne-Mumford topological stacks in [9, Section 14].

Lemma 5.5 Let $\mathcal{X}$ be a topological stack that can be covered by open substacks of the from $[X / G]$, where $G$ is a topological group acting on $X$ with slice property. Then $\mathcal{X}$ has the slice property.

Proof Obvious.

\subsection{Examples of stacks with the slice property}

We list some general classes of group actions with the slice property.

(1) The continuous action of a finite group on a topological space has the slice property.

(2) Let $G$ be a Lie group (not necessarily compact) acting on a topological space $X$. Assume that $X$ is a Cartan $G$-space in the sense of [10, Definition 1.1.2]. Then the action has slice property [10, Theorem 2.3.3]. We recall from [10] that $X$ is called a Cartan $G$-space if for every point of $X$ there is an open neighborhood $U$ such that the set $\{g \in G \mid g U \cap U \neq \varnothing\}$ has compact closure. For instance, if $X$ is locally compact and the action is proper (ie, $G \times X \rightarrow X \times X$ is a proper map), then $X$ is a Cartan $G$-space. Also, if $X$ is completely regular and $G$ compact Lie, then $X$ is a Cartan $G$-space.

The following proposition is immediate.

Proposition 5.6 Let $\mathcal{X}$ be a topological stack (which can be covered by open substacks) of the form $[X / G]$ with $X$ a $C \operatorname{artan} G$-space. Then $\mathcal{X}$ has the slice property. 
Lemma 5.7 Let $[R \rightrightarrows X]$ be a topological groupoid. Assume that the source map $s: R \rightarrow X$ is open and has the property that for every open $V \subseteq R$ the induced map $\left.s\right|_{V}: V \rightarrow s(V)$ admits local sections. Then for every open $U \subseteq X$, the induced map $\left[U /\left.R\right|_{U}\right] \rightarrow[X / R]$ is an open embedding. Here, $\left[\left.R\right|_{U} \rightrightarrows U\right]$ stands for the restriction of $R$ to $U$, which is defined by $\left.R\right|_{U}=(U \times U) \times_{X \times X} R$.

Proof The map $\left[U /\left.R\right|_{U}\right] \rightarrow[X / R]$ is always a monomorphism (ie, fully faithful), without any assumptions on the source map $s$. The extra assumption on $s$ implies that $\left[U /\left.R\right|_{U}\right]$ is equivalent to the open substack $\left[\mathcal{O}(U) /\left.R\right|_{\mathcal{O}(U)}\right]$ of $[X / R]$, where $\mathcal{O}(U)=t\left(s^{-1}(U)\right)$ is the orbit of $U$ (which is open).

Every groupoid $[R \rightrightarrows X]$ in which the source map $s: R \rightarrow X$ is locally isomorphic to the projection map $Y \times X \rightarrow X$ of a product has the property required in Lemma 5.7. These include action groupoids of topological groups acting continuously on topological spaces. Lie groupoids also have this property.

Proposition 5.8 Let $\mathcal{X}=[X / R]$, where $[R \rightrightarrows X]$ is a proper Lie groupoid. Then $\mathcal{X}$ has the slice property.

Proof Let $x \in X$ be an arbitrary point. By [13, Proposition 2.4], the orbit $\mathcal{O}(x)$ is a closed submanifold of $X$. Choose a small enough transversal $\Sigma$ to the orbit $\mathcal{O}(x)$ at $x$. The map $\left.t\right|_{s^{-1} \Sigma}: s^{-1} \Sigma \rightarrow X$ is a submersion, so the quotient stack of the restriction groupoid $\left[\left.R\right|_{S^{-1} \Sigma} \rightrightarrows \Sigma\right]$ is an open substack of $\mathcal{X}$ by Lemma 5.7. Now, $\left[\left.R\right|_{S^{-1} \Sigma} \rightrightarrows \Sigma\right]$ is a Lie groupoid that has $x$ as a fixed point. So, by [14, Theorem 2.3], we can shrink $\Sigma$ (as small as we want) and assume that $\left[\left.R\right|_{s^{-1} \Sigma} \rightrightarrows \Sigma\right]$ is isomorphic to a (linear) action groupoid of the stabilizer group $I_{x}$.

\section{Strongly locally path-connected topological stacks}

We begin with a definition.

Definition 6.1 A topological stack $\mathcal{X}$ is strongly locally path-connected if it has the slice property and, furthermore, the topological spaces $V$ of Definition 5.4 can be chosen to be locally path-connected.

Lemma 6.2 Let $\mathcal{X}$ be a locally path-connected topological stack with the slice property. Assume that for every $x \in \mathcal{X}$ the inertia group $I_{x}$ is locally path-connected. Then $\mathcal{X}$ is strongly locally path-connected. 
Proof We may assume that $\mathcal{X}=[X / G]$, where $G$ is locally path-connected. Since $\mathcal{X}$ is locally path-connected, there is an atlas $p: Y \rightarrow \mathcal{X}$ such that $Y$ is locally pathconnected. Set $Z=Y \times \mathcal{X} X$. Then $Z$ is a $G$-torsor over $Y$. Since $Y$ and $G$ are both locally path-connected, so is $Z$. It follows from Lemma 3.3 that $X$ is locally path-connected.

Proposition 6.3 Let $\mathcal{X}$ be a locally path-connected topological stack. Assume that either of the following holds:

(i) $\mathcal{X}$ is locally isomorphic to a quotient stack $[X / G]$ with $G$ a Lie group and $X$ a Cartan $G$-space;

(ii) $\mathcal{X}$ is the quotient stack of a proper Lie groupoid.

Then $\mathcal{X}$ is strongly locally path-connected.

Proof Use Propositions 5.6, 5.8 and Lemma 6.2.

Recall from Example 2 of Section 5.1 that the Cartan condition is automatically satisfied if any one of the following is true: 1) $G$ is finite; 2) $G$ is compact Lie and $X$ is completely regular; 3) $G$ is an arbitrary Lie group, $X$ is locally compact, and the action is proper, ie, $G \times X \rightarrow X \times X$ is a proper map.

Proposition 6.4 Let $p: \mathcal{Y} \rightarrow \mathcal{X}$ be a covering map of topological stacks, and assume that $\mathcal{X}$ is strongly locally path-connected. Then for every point $x \in \mathcal{X}$, there exists an open substack $x \in \mathcal{U} \subseteq \mathcal{X}$ with the following properties:

(i) $\mathcal{U} \cong\left[U / I_{x}\right]$, where $U$ is a locally path-connected topological space with an action of $I_{x}$ that fixes the (unique) lift of $x$ to $U$ (which we denote again by $x$ ) and has the slice property at $x$;

(ii) $p^{-1}(\mathcal{U}) \cong \bigsqcup_{k \in K}\left[U / H_{k}\right]$, where $H_{k}$, for $k$ ranging in some index set $K$, are open-closed subgroups of $I_{x}$ acting on $U$ via $I_{x}$.

In particular, $\mathcal{Y}$ is also strongly locally path-connected.

Proof Denote $I_{x}$ by $G$ throughout the proof.

By shrinking $\mathcal{X}$ around $x$ we may assume that $\mathcal{X}=[X / G]$, where $G$ and $X$ satisfy (i). Consider the corresponding atlas $X \rightarrow \mathcal{X}$, and let $Y \rightarrow \mathcal{Y}$ be the pullback atlas for $\mathcal{Y}$. The map $q: Y \rightarrow X$, being a pull back of $p$, is again a covering map. There is an open set $U \subseteq X$ containing $x$ over which $q$ trivializes. After replacing $U$ with a smaller open set containing $x$ (say, by the connected component of $x$ in $U$ ), we may assume 
that $U$ is $G$-invariant and path-connected. Set $\mathcal{U}=[U / G]$. We claim that $\mathcal{U}$ has the desired property.

Let $\mathcal{V}=p^{-1}(\mathcal{U}) \subseteq \mathcal{Y}$, and $V=q^{-1}(U) \subseteq Y$. Then $V$ is an atlas for $\mathcal{V}$ and is of the form

$$
V=\coprod_{j \in J} U_{j}, U_{j}=U
$$

for some index set $J$. Set $R=V \times \mathcal{V} V$ (so $\mathcal{V} \cong[V / R]$ ). Note that $V$ can be viewed as an atlas for $\mathcal{U}$ too, and if we set $R^{\prime}=V \times \mathcal{U} V$, then the groupoid $[R \rightrightarrows V]$ is an open-closed subgroupoid of $\left[R^{\prime} \rightrightarrows V\right]$ (Corollary 3.6). Observe that, as a topological space, $R^{\prime}$ is homeomorphic to a disjoint union of $J \times J$ copies of $G \times U$, and the restriction of $s: R^{\prime} \rightarrow V$ and $t: R^{\prime} \rightarrow V$ to each of these copies factors through some $U_{j} \subset V$ via a map that is isomorphic to the projection $G \times U \rightarrow U$. In particular, $s: R^{\prime} \rightarrow V$ (and also $t$ ) has the following two properties: 1) For every $W \subseteq R^{\prime}, s(W)$ is open in $V$ and the restriction $\left.s\right|_{W}: W \rightarrow s(W)$ admits local sections; 2) If $W$ is also closed, then $s(W)$ is a disjoint union of copies of $U$ in $V=\bigsqcup U_{j}$.

Now, observe that, since $[R \rightrightarrows V]$ is an open-closed subgroupoid of $\left[R^{\prime} \rightrightarrows V\right]$, properties (1) and (2) mentioned above also hold for $s, t: R \rightarrow V$. An immediate consequence is that, for each $U_{j} \subseteq V=\bigsqcup U_{j}$, the orbit $\mathcal{O}\left(U_{j}\right)$ is a disjoint union of copies of $U$ in $V$; in particular $\mathcal{O}\left(U_{j}\right) \subseteq V$ is open-closed. Therefore, by Lemma 5.7, $\left[U_{j} /\left.R\right|_{U_{j}}\right]$ is an open-closed substack of $\mathcal{V}$.

Let us analyze what $\left[U_{j} /\left.R\right|_{U_{j}}\right]$ looks like. Recall that $\left.R\right|_{U_{j}}=\left(U_{j} \times U_{j}\right) \times_{V \times V} R$. Equivalently, $\left.R\right|_{U_{j}}=s^{-1}\left(U_{j}\right) \cap t^{-1}\left(U_{j}\right)$. Note that $s^{-1}\left(U_{j}\right) \cong G \times U$. Hence, since $U$ is connected, $s^{-1}\left(U_{j}\right) \cap t^{-1}\left(U_{j}\right)$, being an open-closed subspace of $s^{-1}\left(U_{j}\right) \cong G \times U$, is of the form $H \times U$, where $H \subseteq G$ is an open-closed subspace. It is easy to see that $H$ is in fact a subgroup of $G$, so that the groupoid $\left[U_{j} /\left.R\right|_{U_{j}}\right]$ is simply (isomorphic to) the action groupoid of $H$ acting on $U$ via $G$.

So, we have shown that $\mathcal{V}$ is a disjoint union of open-closed substacks of the form $\left[U_{j} /\left.R\right|_{U_{j}}\right]$ each of which is equivalent to $[U / H]$ for some open-closed subgroup $H \subseteq G$. (Note that different $j$ 's may correspond to the same substack $\left[U_{j} /\left.R\right|_{U_{j}}\right]$.) The proof is complete.

\section{Relation between various Galois theories}

In this section, we take a closer look at the Galois categories introduced in Section 4.1Section 4.4 and study the relations between them. The most important class for us is Free. In the next section, we interpret these results in terms of fundamental groups of stacks and their coarse moduli spaces. 


\subsection{Relation between Free $_{\mathcal{X}}$ and Full $_{\mathcal{X}_{\bmod }}$}

Lemma 7.1 Let $\mathcal{X}$ be a topological stack, and let $\pi: \mathcal{X} \rightarrow \mathcal{X}_{\text {mod }}$ be its moduli map. Let $Y$ be a topological space. Let $Y \rightarrow \mathcal{X}_{\text {mod }}$ be a local homeomorphism, and set $\mathcal{Y}:=Y \times \mathcal{X}_{\text {mod }} \mathcal{X}_{\text {mod }}$. Then $Y$ is the coarse moduli space of $\mathcal{Y}$. That is, the induced map $g: \mathcal{Y}_{\text {mod }} \rightarrow Y$ is a homeomorphism.

Proof By the very definition of the coarse moduli space, the statement is local on $Y$. That is, it is enough to prove the statement after replacing $Y$ by an open covering. So, we may assume that $Y \rightarrow \mathcal{X}_{\text {mod }}$ is a disjoint union of open embedding, in which case the lemma is obvious.

Lemma 7.2 Let $\mathcal{X}$ be a topological stack, and let $p: \mathcal{X} \rightarrow A$ be an arbitrary map to a topological space $A$. Let $g: B \rightarrow A$ be a covering map of topological spaces. Then the induced map $f: \mathcal{X} \times_{A} B \rightarrow \mathcal{X}$ is free (Section 4.4).

Proof Denote $\mathcal{X} \times{ }_{A} B$ by $\mathcal{Y}$. We have to show that $f$ is FPR and that the induced map $f_{\text {mod }}: \mathcal{Y}_{\text {mod }} \rightarrow \mathcal{X}_{\text {mod }}$ is a covering map.

Proof that $f$ is FPR Choose an atlas $p: X \rightarrow \mathcal{X}$, and let $q: Y \rightarrow \mathcal{Y}$ be the pullback atlas for $\mathcal{Y}$. Denote the corresponding groupoids by $\left[R_{X} \rightrightarrows X\right]$ and $\left[R_{Y} \rightrightarrows Y\right]$. Let $S_{X} \rightarrow X$ and $S_{Y} \rightarrow Y$ be the relative stabilizer groups of these groupoids (that is, $S_{X}=X \times_{X \times{ }_{A} X} R$, where $X \rightarrow X \times_{A} X$ is the diagonal). Observe that, for every point $x \in X$, the fiber $S_{x}$ of $R \rightarrow X \times_{A} X$ over the point $(x, x)$ is naturally isomorphic to the inertia group $I_{p(x)}$. The result now follows from the standard fact that the diagram

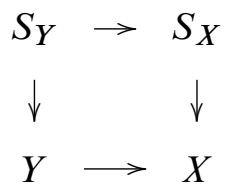

is cartesian.

Proof that $f_{\text {mod }}$ is a covering map The map $p$ factors through the moduli map $\pi: \mathcal{X} \rightarrow \mathcal{X}_{\text {mod }}$. Set $Y^{\prime}:=B \times_{A} \mathcal{X}_{\text {mod }}$. Then $Y^{\prime} \rightarrow \mathcal{X}_{\text {mod }}$ is a covering map. Using the fact that the diagram

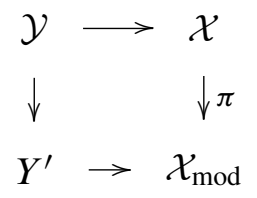

Algebraic 8 Geometric Topology, Volume 8 (2008) 
is cartesian, together with the fact that taking coarse moduli space commutes with base extension along covering maps (Lemma 7.1), we see that $Y^{\prime}$ is naturally homeomorphic to $\mathcal{Y}_{\text {mod }}$, and the map $Y^{\prime} \rightarrow \mathcal{X}_{\text {mod }}$ is naturally identified with $f_{\text {mod }}$.

Lemma 7.3 Let $f: \mathcal{Y} \rightarrow \mathcal{X}$ be a free covering stack. Then the following diagram is cartesian:

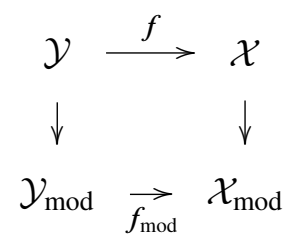

Proof Set $\mathcal{Y}^{\prime}:=\mathcal{Y}_{\text {mod }} \times \mathcal{X}_{\text {mod }} \mathcal{X}$. Then $\mathcal{Y}^{\prime} \rightarrow \mathcal{X}$ is a covering stack of $\mathcal{X}$, and there is a natural map $\mathcal{Y} \rightarrow \mathcal{Y}^{\prime}$ of covering stacks over $\mathcal{X}$. This maps induces a bijection on the fibers, so it is an isomorphism.

Using the above three lemmas, the following proposition is immediate.

Proposition 7.4 Let $\mathcal{X}$ be a topological stack. Then there is an equivalence of categories

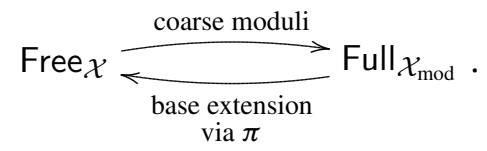

The similar statement is true for connected covering stacks. Finally, the statement remains valid if we add the adjective "pointed".

\subsection{Description of FPR}

The next proposition leads to a satisfactory description of the Galois category FPR. Recall from [9, Section 17] that, for every $x \in \mathcal{X}$, there is a natural group homomorphism $\omega_{x}: I_{x} \rightarrow \pi_{1}(\mathcal{X}, x)$.

Lemma 7.5 Let $(\mathcal{X}, x)$ be a pointed connected topological stack, and let $f:(\mathcal{Y}, y) \rightarrow$ $(\mathcal{X}, x)$ be a pointed connected covering map. Then we have the following:

(i) $f$ is FPR at $y$ if and only if the corresponding subgroup $H \subseteq \pi_{1}(\mathcal{X}, x)$ contains $\omega_{x}\left(I_{x}\right)$;

(ii) For every point $x^{\prime}$, and every path $\gamma$ in $\mathcal{X}$ from $x^{\prime}$ to $x$, identify $\omega_{x^{\prime}}\left(I_{x^{\prime}}\right) \subseteq$ $\pi_{1}\left(\mathcal{X}, x^{\prime}\right)$ with a subgroup of $\pi_{1}(\mathcal{X}, x)$ via the isomorphism $\gamma_{*}: \pi_{1}\left(\mathcal{X}, x^{\prime}\right) \rightarrow$ $\pi_{1}(\mathcal{X}, x)$. Let $N$ be the (necessarily normal) subgroup of $\pi_{1}(\mathcal{X}, x)$ generated by all these groups. Then $f$ is FPR if and only if the corresponding subgroup $H \subseteq \pi_{1}(\mathcal{X}, x)$ contains $N$. 
Proof (i) By Lemma 18.16 of [9], we have a cartesian diagram:

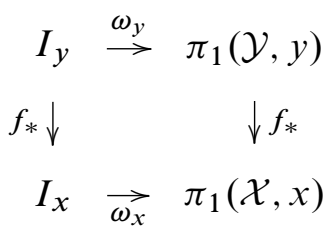

So, the map $f_{*}: I_{y} \rightarrow I_{x}$ (which is already injective because $f$ is representable) is an isomorphism if and only if the image of $f_{*}: \pi_{1}(\mathcal{Y}, y) \rightarrow \pi_{1}(\mathcal{X}, x)$, namely $H$, contains $I_{x}$.

(ii) Lift $\gamma$ to a path in $\mathcal{Y}$ ending at $y$, and call the starting point $y^{\prime}$. Then $f$ is FPR at $y^{\prime}$ if and only if $H$ contains $\gamma_{*}\left(\omega_{x^{\prime}}\left(I_{x^{\prime}}\right)\right)$.

Let us rephrase part (ii) of the above lemma as a proposition.

Proposition 7.6 The open subgroups of the FPR topology on $\pi_{1}(\mathcal{X}, x)$ are precisely the open subgroups of $\pi_{1}(\mathcal{X}, x)$ in the full topology which contain $N$.

\subsection{Relation between Free and FPR}

The subcategory Free $\subseteq$ FPR is not as easy to describe in general. But we have the following results.

Proposition 7.7 If $\mathcal{X}$ is a strongly locally path-connected topological stack, then every FPR covering stack is automatically free. That is, Free $=$ FPR.

Proof This follows immediately from Proposition 6.4 .

When $\mathcal{X}$ is not strongly locally path-connected, we could still say something.

Proposition 7.8 Let $[R \rightrightarrows X]$ be a topological groupoid, and let $\mathcal{X}=[X / R]$ be its quotient stack. Assume that either of the following holds:

(i) $X$ is metrizable and $s, t: R \rightarrow X$ are closed maps;

(ii) $X$ is Hausdorff and $s, t: R \rightarrow X$ are proper.

Then every finite covering stack of $\mathcal{X}$ that is FPR is automatically free. That is, FPR $\cap$ Fin $=$ Free $\cap$ Fin .

Algebraic 8 Geometric Topology, Volume 8 (2008) 
Proof We may assume that $\mathcal{X}$ is connected. Let $f: \mathcal{Y} \rightarrow \mathcal{X}$ be a connected finite FPR covering stack of degree $n$. We have to show that $f_{\text {mod }}: \mathcal{Y}_{\text {mod }} \rightarrow \mathcal{X}_{\text {mod }}$ is also a covering map.

Consider the atlas $p: X \rightarrow \mathcal{X}$, and let $q: Y \rightarrow \mathcal{Y}$ be the pullback atlas for $\mathcal{Y}$. Let $\left[R_{Y} \rightrightarrows Y\right]$ be the corresponding groupoid. To have consistent notation, we denote $R$ by $R_{X}$. The map $g: Y \rightarrow X$ is a covering map of degree $n$. Recall [9, Example 4.13] that $\mathcal{X}_{\text {mod }}$ is homeomorphic to the coarse quotient of $X$ by the equivalence relation induced from $R_{X}$ (and similarly for $\mathcal{Y}_{\text {mod }}$ ). We have a commutative diagram:

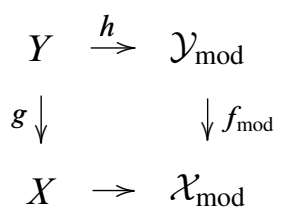

By Lemma 4.3, both vertical maps have constant degree $n$. Therefore, $h$ is a fiberwise bijection. Take a point $\bar{x} \in \mathcal{X}_{\text {mod }}$, and pick a lift $x \in X$ for it. Let $\bar{y}_{1}, \cdots, \bar{y}_{n} \in \mathcal{Y}_{\text {mod }}$ be the elements of the fiber of $f_{\text {mod }}$ over $\bar{x}$. Similarly, let $y_{1}, \cdots, y_{n} \in Y$ be the elements of the fiber of $g$ over $x$. Consider the orbit $B_{i}:=\mathcal{O}\left(y_{i}\right) \subseteq Y$ of $y_{i}$ under the action of the groupoid $\left[R_{Y} \rightrightarrows Y\right]$ (this is simply the fiber of $h: Y \rightarrow \mathcal{Y}_{\text {mod }}$ over $y_{i}$ ). By hypothesis, $B_{i}$ is closed. Since $h$ is a fiberwise bijection, the restriction $\left.g\right|_{B_{i}}: B_{i} \rightarrow X$ is injective for every $i$. It is also closed, because $g$ is a finite cover. Therefore, $\left.g\right|_{B_{i}}: B_{i} \rightarrow A$ is a homeomorphism for every $i$, where $A=g\left(B_{1}\right)=\cdots=g\left(B_{n}\right)=\mathcal{O}(x)$. In other words, the covering map $g: Y \rightarrow X$ trivializes over $A \subseteq X$. We claim that there exists an open $A \subseteq U$ such that $g$ trivializes over $U$ as well. By condition (i) or (ii), we can find open sets $B_{i} \subseteq V_{i}$ such that $V_{i} \cap V_{j}=\varnothing$, for every $i \neq j$ (see Lemma 7.9 below). By shrinking each $V_{i}$, we may assume that $\left.g\right|_{V_{i}}: V_{i} \rightarrow U$ is a homeomorphism for every $i$. It is easy to check that $U:=\cap g\left(V_{i}\right)$ has the desired property.

The next claim is that, after some more shrinking, we may assume that $U$ and $V_{i}$, $i=1, \cdots, n$, are invariant open sets for the corresponding groupoids. To do so, note that the source and target maps of the groupoids $\left[R_{X} \rightrightarrows X\right]$ and $\left[R_{Y} \rightrightarrows Y\right]$ are both closed maps. This follows from the hypothesis and the fact that the diagram

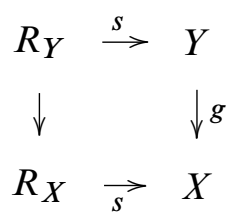

Algebraic 83 Geometric Topology, Volume 8 (2008) 
is cartesian. So, we may replace $U$ by $U-s\left(R_{X}-t^{-1}(U)\right)$, and similarly, each $V_{i}$ by $V_{i}-s\left(R_{Y}-t^{-1}\left(V_{i}\right)\right)$. Note that we still have $A \subseteq U, B_{i} \subseteq V_{i}$, and each $V_{i}$ maps homeomorphically to $U$. Hence, after passing to the coarse moduli spaces, we obtain an open neighborhood of $\bar{U}$ of $\bar{x}$ over which $f_{\text {mod }}$ trivializes as an $n$-sheeted covering (the sheets being $\bar{V}_{1}, \cdots, \bar{V}_{n}$ ). The proof is complete.

Lemma 7.9 Let $f: Y \rightarrow X$ be a finite covering map of topological spaces. If $X$ is metrizable, then so is $Y$

Proof This follows from the Smirnov Metrization Theorem which says that a topological space $X$ is metrizable if and only if it is paracompact, Hausdorff and locally metrizable. All these properties are easily seen to be stable under passing to finite covering spaces.

For the reader who is not familiar with this version of the Smirnov Metrization Theorem, we provide a quick proof. If $X$ is metrizable, it is clearly locally metrizable and Hausdorff. Paracompactness of $X$ is Stone's Theorem; see Nagata [8, page 153, Corollary 1]. The converse statement follows from the corollary on page 212 of [8].

\subsection{Description of Full and Fin when $\mathcal{X}$ is semilocally 1-connected}

When $\mathcal{X}$ is semilocally 1 -connected things are as nice as they can be, because the (pointed) covering stacks of $\mathcal{X}$ are in a bijection with subgroups of $\pi_{1}(\mathcal{X}, x)$; see Section 18.2 of [9].

Proposition 7.10 Suppose that $\mathcal{X}$ is a connected, locally path-connected, semilocally 1 -connected topological stack. Then Full corresponds to the discrete topology on $\pi_{1}(\mathcal{X}, x)$ and Fin corresponds to the profinite topology. We have $\pi_{1}^{\prime}(\mathcal{X}, x)=\pi_{1}(\mathcal{X}, x)$, and $\pi_{1}(\mathcal{X}, x)_{\mathrm{Fin}}^{\wedge}=\widehat{\pi_{1}(\mathcal{X}, x)}$, the profinite completion of $\pi_{1}(\mathcal{X}, x)$.

Proof The statement about Full follows from Proposition 2.9. The second statement is obvious.

\section{Fundamental group of the coarse moduli space}

In this section, we translate the results of the previous section in terms of the fundamental groups. The outcome is some formulas for the fundamental group of the coarse moduli space of a topological stack.

Notation Throughout this section, the group $N \subseteq \pi_{1}(\mathcal{X}, x)$ refers to the group defined in Lemma 7.5. 
Proposition 8.1 Let $\mathcal{X}$ be a connected locally path-connected topological stack. Then we have the following:

(i) The image of the natural map $\pi_{*}^{\prime}: \pi_{1}^{\prime}(\mathcal{X}, x) \rightarrow \pi_{1}^{\prime}\left(\mathcal{X}_{\text {mod }}, x\right)$ is not contained in any proper open subgroup of $\pi_{1}^{\prime}\left(\mathcal{X}_{\bmod }, x\right)$. In particular, if the full topology on $\pi_{1}\left(\mathcal{X}_{\text {mod }}, x\right)$ has a basis of open neighborhoods (equivalently, every covering space of $\mathcal{X}_{\text {mod }}$ can be dominated by a Galois covering), then $\pi_{*}^{\prime}$ has a dense image.

(ii) There is a natural isomorphism

$$
\pi_{1}(\mathcal{X}, x) \stackrel{\wedge}{\wedge} \stackrel{\sim}{\longrightarrow} \pi_{1}^{\prime}\left(\mathcal{X}_{\text {mod }}, x\right) .
$$

(iii) If $\mathcal{X}$ is strongly locally path-connected, then we have a natural isomorphism

$$
\left(\pi_{1}(\mathcal{X}, x) / N\right)^{\prime} \stackrel{\sim}{\longrightarrow} \pi_{1}^{\prime}\left(\mathcal{X}_{\bmod }, x\right) .
$$

(The left hand side is the completion with respect to the quotient topology induced on $\pi_{1}(\mathcal{X}, x) / N$ from the full topology of $\pi_{1}(\mathcal{X}, x)$.)

(iv) If $\mathcal{X}$ is as in Proposition 7.8, then we have a natural isomorphism

$$
\left(\pi_{1}(\mathcal{X}, x) / N\right)_{\mathrm{Fin}}^{\wedge} \stackrel{\sim}{\longrightarrow} \pi_{1}\left(\mathcal{X}_{\mathrm{mod}}, x\right)_{\mathrm{Fin}}^{\wedge} .
$$

Proof (i) This is equivalent to saying that the pullback via $\pi: \mathcal{X} \rightarrow \mathcal{X}_{\text {mod }}$ of a connected covering space $Y$ of $\mathcal{X}_{\text {mod }}$ remains connected. Denote this pullback by $\mathcal{Y}$. Since $\mathcal{X}$ is locally connected, so is $\mathcal{Y}$. If $\mathcal{Y}$ is not connected, we can write it as a disjoint union of two open-closed substacks $\mathcal{Y}_{1} \amalg \mathcal{Y}_{2}$. But then, by Lemma 7.1, we would have a decomposition $\mathcal{Y}_{1 \text {, mod }} \bigsqcup \mathcal{Y}_{2 \text {,mod }}$ of $Y$ into open-closed subspaces, which is impossible.

(ii) This follows from Proposition 7.4.

(iii) This follows from Part (ii) and Proposition 7.6 (also see Example 2.10(1)).

(iv) This follows from Proposition 7.8.

In general, it is desirable to work with the actual fundamental group $\pi_{1}(\mathcal{X}, x)$ (ie, the one defined using loops) rather than the fancy prodiscrete fundamental groups of Section 4.1. But to do so one needs to assume that $\mathcal{X}$ is semilocally 1 -connected. In what follows we analyze what happens in the presence of this condition. Recall from Section 7.4 that in this case the full topology on $\pi_{1}(\mathcal{X}, x)$ is discrete.

Corollary 8.2 Let $\mathcal{X}$ be a connected locally path-connected topological stack. Assume that $\mathcal{X}_{\text {mod }}$ is semilocally 1 -connected. Then the map $\pi_{*}: \pi_{1}(\mathcal{X}, x) \rightarrow \pi_{1}\left(\mathcal{X}_{\text {mod }}, x\right)$ is surjective. 
Proof 2 Let $H \subseteq \pi_{1}\left(\mathcal{X}_{\text {mod }}, x\right)$ be the image of $\pi_{*}: \pi_{1}(\mathcal{X}, x) \rightarrow \pi_{1}\left(\mathcal{X}_{\text {mod }}, x\right)$. Since $\pi_{1}(\mathcal{X}, x) \rightarrow H$ is continuous, there is a unique extension $\pi_{1}^{\prime}(\mathcal{X}, x) \rightarrow H$, by Corollary 2.11. This extension coincides with $\pi_{*}^{\prime}$ of Proposition 8.1(i). Therefore, $H$ must be dense in $\pi_{1}\left(\mathcal{X}_{\text {mod }}, x\right)$. But $\pi_{1}\left(\mathcal{X}_{\text {mod }}, x\right)$ is discrete by Proposition 7.10. So $H=$ $\pi_{1}\left(\mathcal{X}_{\text {mod }}, x\right)$.

Theorem 8.3 Let $(\mathcal{X}, x)$ be a connected topological stack. Assume that $\mathcal{X}$ and $\mathcal{X}_{\text {mod }}$ are semilocally 1 -connected.

(i) If $\mathcal{X}$ is strongly locally path-connected, then we have a natural isomorphism

$$
\pi_{1}(\mathcal{X}, x) / N \stackrel{\sim}{\longrightarrow} \pi_{1}\left(\mathcal{X}_{\text {mod }}, x\right) .
$$

(ii) Assume that $\mathcal{X}$ is the quotient stack of a topological groupoid $[R \rightrightarrows X]$ in which either $X$ is metrizable and the source map $s: R \rightarrow X$ is closed, or $X$ is Hausdorff and the source map $s: R \rightarrow X$ is proper. Then we have a natural isomorphism

$$
\widehat{\pi_{1}(\mathcal{X}, x) / N} \stackrel{\sim}{\longrightarrow} \overline{\pi_{1}\left(\mathcal{X}_{\bmod }, x\right)},
$$

where ${ }^{\wedge}$ stands for profinite completion. (The ${ }^{\wedge}$ on the left hand side is over the whole expression.)

Proof Use Proposition 8.1(iii)-(iv) and Proposition 7.10.

Remark 8.4 It is easy to see [9, Lemma 18.4] that, if $\mathcal{X}$ is a topological stack which is (locally) path-connected, then so is $\mathcal{X}_{\text {mod }}$. The similar statement is not true for semilocally 1 -connected stacks in general. But it is true when $\mathcal{X}=[X / G]$, where $G$ is a Lie group and $X$ is a Cartan $G$-space. This follows from [10, Theorem 2.3.3]. In the case where $G$ is a compact Lie group this is Corollary 6.4 in Chapter II of [4].

Example 8.5 Let $\mathcal{G}$ be a graph-of-groups. It is shown in [9] that $\mathcal{G}$ can be realized as a Deligne-Mumford topological stack. For each vertex $v$ of $\mathcal{G}$, let $G_{v}$ denote the corresponding group. Then we have $G_{v}=I_{v}$, and the homomorphism $G_{v} \rightarrow \pi_{1}(\mathcal{G}, v)$ defined by Serre coincides with our map $\omega_{v}: I_{v} \rightarrow \pi_{1}(\mathcal{G}, v)$. The similar thing is true for the homomorphisms $G_{e} \rightarrow \pi_{1}\left(\mathcal{G}, e_{0}\right)$, where $e_{0}$ is any point on the edge $e$.

If we let $N$ be the normal subgroup generated by the images of all $G_{v}$ and $G_{e}$ in $\pi_{1}(\mathcal{G})$, we find that the fundamental group of the underlying graph of $\mathcal{G}$ is isomorphic to $\pi_{1}(\mathcal{G}) / N$. This is of course a well-known formula (see Bass [3, Example 2.14]). 
The similar result is valid for complexes-of-groups as well, and this should presumably be well known too.

Example 8.6 Let $X$ and $Y$ be as in Example 4.1. There is a shift action of $\mathbb{Z}$ on $X$ and $Y$. Set $\mathcal{X}=[X / \mathbb{Z}]$ and $\mathcal{Y}=[Y / \mathbb{Z}]$. The coarse moduli space of $\mathcal{Y}$ is $Y / \mathbb{Z}$, which is $S^{1}$ (with the usual topology). The coarse moduli space of $\mathcal{X}$ is $S^{1}$ with a topology that is the usual topology away from the base point $\bullet \in S^{1}$, but the only open containing $\bullet$ is the entire space; this is a contractible space.

The fundamental group of $Y$ is $\mathbb{F}^{\mathbb{Z}}=\left\langle a_{i} \mid i \in \mathbb{Z}\right\rangle$. The fundamental group of $X$ contains $\mathbb{F}^{\mathbb{Z}}$, but it is considerably bigger (it is indeed uncountable). The $\mathbb{Z}$-actions on $X$ and $Y$ induce $\mathbb{Z}$-actions on $\pi_{1}(X)$ and $\pi_{1}(Y)$. In the case of $Y$, this action is simply given by shifting the generators. In the case of $X$, it is also some sort of a shift, but it is more complicated to describe. We have

$$
\pi_{1}(\mathcal{Y}) \cong \mathbb{F}^{\mathbb{Z}} \rtimes \mathbb{Z} \text {, and } \pi_{1}(\mathcal{X}) \cong \pi_{1}(X) \rtimes \mathbb{Z} .
$$

The image of the map $\omega_{\bullet}: \mathbb{Z}=I_{\bullet} \rightarrow \pi_{1}(\mathcal{Y}, \bullet)$ is simply the factor $\mathbb{Z}$ of the semidirect product (similarly for $\pi_{1}(\mathcal{X})$ ). If we kill this subgroup of $\pi_{1}(\mathcal{Y})$ we get a group that is isomorphic to $\mathbb{Z} \cong \pi_{1}\left(\mathcal{X}_{\text {mod }}\right)$, as predicted by Theorem 8.3. (In fact, it seems that the same is true for $\mathcal{Y}$. That is, if we kill $\mathbb{Z}$ in $\pi_{1}(\mathcal{X})$ we end up with the trivial group. Note that this is not predicted by Theorem 8.3 , as $\mathcal{Y}$ is not semilocally 1 -connected.)

\section{Application to quotients of group actions: the exact sequence}

Results of the previous section can be used to compute the fundamental group of the coarse quotient space of a topological group action. The idea is that the fundamental group of the quotient stack $[X / G]$ of a group action is easy to compute thanks to the fiber homotopy exact sequence of the fibration $G \rightarrow X \rightarrow[X / G]$. Theorem 8.3 then can be used to compute the fundamental group of $[X / G]_{\bmod }=X / G$ from the fixed point data of the action (which manifests itself in the inertia groups of $[X / G]$ ).

Conventions In this and the next sections, $G$ will be a topological group acting continuously on a connected, locally path-connected, semilocally 1 -connected topological space $X$. We assume that the coarse quotient $X / G$ is also semilocally 1 -connected; see Remark 8.4. We denote the group of path components of $G$ by $\mathbf{G}$. We let $\mathbf{I} \subset \mathbf{G}$ be the subgroup generated by the set of elements of those path components of $G$ which contain at least one element $g$ such that the action of $g$ on $X$ has a fixed point. 
Theorem 9.1 Let $G$ and $X$ be as above. Suppose that the action has slice property. Assume that all stabilizer groups $I_{x}$ of the action are locally path-connected. Fix a base point $x$ in $X$ and let $\bar{x}$ be its image in $X / G$. Then we have an exact sequence

$$
\pi_{1}(X, x) \rightarrow \pi_{1}(X / G, \bar{x}) \rightarrow \mathbf{G} / \mathbf{I} \rightarrow 1 .
$$

In particular, if $X$ is simply connected, then we have an isomorphism

$$
\pi_{1}(X / G) \stackrel{\sim}{\longrightarrow} \mathbf{G} / \mathbf{I} .
$$

Proof Set $\mathcal{X}=[X / G]$. By abuse of notation we denote the image of $x$ in $[X / G]$ also by $\bar{x}$.

By Lemma $6.2, \mathcal{X}$ is a strongly locally path-connected topological stack. Hence, we can apply Theorem 8.3(i). To get the desired exact sequence, we computed the group $N$ appearing in Theorem 8.3.

Since $p$ is a Serre fibration with fiber $G$, we have a fiber homotopy exact sequence

$$
\pi_{1}(X, x) \rightarrow \pi_{1}(\mathcal{X}, \bar{x}) \rightarrow \mathbf{G} \rightarrow 1 .
$$

It is enough to show that the image of $N$ in $\mathbf{G}$ is equal to $\mathbf{I}$.

We have a natural isomorphism $\varphi: I_{\bar{x}} \stackrel{\sim}{\longrightarrow} I_{x}$, where $I_{x} \subseteq G$ is the stabilizer group of $x$. The claim is that the following diagram is commutative:

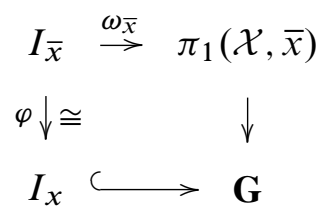

Take an element $\gamma \in I_{\bar{x}}$. Recall that the corresponding loop $\tilde{\gamma}:=\omega_{\bar{x}}(\gamma): S^{1} \rightarrow \mathcal{X}$ is defined by the triple $\left(h, \epsilon_{0}, \epsilon_{1}\right)$, where $h:[0,1] \rightarrow \mathcal{X}$ is the constant path at $\bar{x}, \epsilon_{0}=$ id and $\epsilon_{1}=\gamma$ (see Section 17 of [9] for definitions). We have to show that the image of $\tilde{\gamma}$ in $\mathbf{G}$ is equal to $\varphi(\gamma)$.

Consider the pullback $T:=S^{1} \times_{\mathcal{X}} X$ of $X$ over $S^{1}$, where the map $S^{1} \rightarrow \mathcal{X}$ is the $\tilde{\gamma}$ defined above. Note that $T$ is naturally pointed with the point $*=\left(\bullet, \operatorname{id}_{\bar{x}}, x\right)$ sitting above $\bullet \in S^{1}$. Here, $\operatorname{id}_{\bar{x}}$ is the identity transformation from $\tilde{\gamma}(\bullet)=\bar{x}$ to $p(x)=\bar{x}$.

As a $G$-torsor over $S^{1}, T$ can be described as the quotient of $[0,1] \times G$ by the relation $(1, g) \sim(0, g \varphi(\gamma))$. Under this identification, $*$ corresponds to the point $\left(0,1_{G}\right)$, where $1_{G}$ is the identity element of $G$. 
To find the image of $\tilde{\gamma}$ in $\mathbf{G}$, we have to choose a lift $F$ in diagram

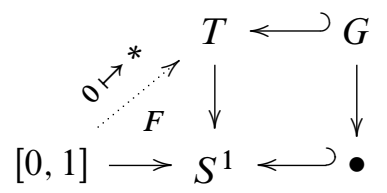

and determine the path component of $F(1) \in G$. But from the description of the torsor $T$, it is clear that $F(1)$ is in the same path component as $\varphi(\gamma) \in G$. This proves the commutativity of the above square.

We can now repeat the same argument for different choices of base points $x^{\prime} \in X$, and then transport the situation over to $x$ by choosing a path connecting $x$ and $x^{\prime}$. This shows that the image of $N$ in $\mathbf{G}$ is $\mathbf{I}$.

Remark 9.2 All the hypotheses of Theorem 9.1 are automatically satisfied if $G$ is a Lie group and $X$ is a connected, locally path-connected, semilocally 1 -connected Cartan $G$-space. See Section 5.1 and Remark 8.4.

Example 9.3 Let $G$ be a compact Lie group acting on $S^{n}$. If $n$ is even, then $S^{n} / G$ is simply connected (Brouwer's fixed point theorem). The same is true if $n$ is arbitrary and $G$ is connected.

Example 9.4 Let $G \subset \mathrm{PSL}_{2}(\mathbb{R})$ be a Fuchsian group. Assume that the fundamental domain of $G$ is not compact. Then $G \cong T \times F$, where $T$ is generated by torsion elements and $F$ is free.

To prove this, note that the action of $G$ on the upper half-plane is properly discontinuous and the coarse quotient of this action is a noncompact Riemann surface. Let $T$ be the subgroup generated by all torsion elements of $G$. By Theorem 9.1, $G / T$ is isomorphic the fundamental group $F$ of this noncompact Riemann surface, hence it is free.

Example 9.5 The weighted projective space $\mathbb{P}\left(n_{0}, \cdots n_{k}\right), k \geq 1$, is defined to be the quotients space $\mathbb{C}^{n+1}-\{0\} / \mathbb{C}^{*}$, where $\lambda \in \mathbb{C}^{*}$ acts on $\mathbb{C}^{n+1}-\{0\}$ via multiplication by $\left(\lambda^{n_{0}}, \cdots, \lambda^{n_{k}}\right)$. It is a standard fact that $\mathbb{P}\left(n_{0}, \cdots n_{k}\right)$ is simply connected. This easily follows from Theorem 9.1. More generally, whenever $G$ is a compact connected Lie group acting on a simply connected completely regular topological space $X$, then $X / G$ is simply connected. Indeed, it is true that the stack quotient $[X / G]$ is simply connected. This follows from the fiber homotopy exact sequence for the fibration $X \rightarrow[X / G]$. 
Theorem 9.6 Assume that $G$ is a compact topological group acting on a Hausdorff space $X$. Then we have an exact sequence

$$
\widehat{\pi_{1}(X)} \rightarrow \widehat{\pi_{1}(X / G)} \rightarrow \widehat{\mathbf{G} / \mathbf{I}} \rightarrow\{*\},
$$

where ${ }^{\wedge}$ stands for profinite completion. In particular, when $X$ is simply connected we have an isomorphism

$$
\widehat{\pi_{1}(X / G)} \stackrel{\sim}{\longrightarrow} \widehat{\mathbf{G} / \mathbf{I}} .
$$

Proof The proof of Theorem 9.1 is valid up to the point that there is an exact sequence

$$
\pi_{1}(X) \rightarrow \pi_{1}(\mathcal{X}) / N \rightarrow \mathbf{G} / \mathbf{I} \rightarrow 1 .
$$

However, we can not say that $\pi_{1}\left(\mathcal{X}_{\text {mod }}\right) \cong \pi_{1}(\mathcal{X}) / N$. But we can say so after passing to profinite completion (Theorem 8.3(ii)). The result now follows from the fact that profinite completion is right exact.

\section{Application to quotients of group actions: the explicit for- mula}

In this section we give a more explicit version of Theorem 9.1. Without loss of generality, we will assume that the action has a global fixed point. Remark 10.7 explains why we can make this assumption.

Construction Let $G$ be a group acting continuously on a topological space $X$. Assume that there is $x \in X$ which is fixed by the entire action. For every triple $(g, y, \gamma)$, with $g \in G, y \in X^{g}$ and $\gamma$ a path from $x$ to $y$, define $\lambda_{g, y, \gamma} \in \pi_{1}(X, x)$ to be the loop $\gamma(g \gamma)^{-1}$. Let $K \subseteq \pi_{1}(X, x)$ be the subgroup generated by all such $\lambda_{g, y, \gamma}$. This is easily seen to be a normal subgroup.

We will abuse the notation and denote the images of $x$ in $[X / G]$ and $X / G$ both by $\bar{x}$.

Theorem 10.1 Let $X$ and $G$ be as in Theorem 9.1 (also see Remark 9.2). Assume that there is $x \in X$ which is fixed by the action. Let $K$ be the subgroup of $\pi_{1}(X, x)$ defined in the previous paragraph. Then we have a natural isomorphism

$$
\pi_{1}(X, x) / K \stackrel{\sim}{\longrightarrow} \pi_{1}(X / G, \bar{x}) .
$$

Proof Set $\mathcal{X}=[X / G]$, and let $p: X \rightarrow \mathcal{X}$ denote the quotient map.

First, we show that $\pi_{1}(\mathcal{X}, \bar{x}) \cong \pi_{1}(X, x) \rtimes \mathbf{G}$. Here, $\mathbf{G}$ stands for the group of path components of $G$, and the action of $\mathbf{G}$ on $\pi_{1}(X, x)$ is the obvious one. The map 
$X \rightarrow \mathcal{X}$ is a Serre fibration with fiber $G$, so it gives rise to the fiber homotopy exact sequence

$$
\pi_{1}(G) \rightarrow \pi_{1}(X, x) \rightarrow \pi_{1}(\mathcal{X}, \bar{x}) \rightarrow \mathbf{G} \rightarrow 1 .
$$

It is easily seen that the leftmost map is the trivial map, so we actually have a short exact sequence

$$
1 \rightarrow \pi_{1}(X, x) \rightarrow \pi_{1}(\mathcal{X}, \bar{x}) \rightarrow \mathbf{G} \rightarrow 1 .
$$

Since $x$ is the fixed point of the entire action, we have $I_{\bar{x}}=G$. The map $\omega_{\bar{x}}: G=$ $I_{\bar{x}} \rightarrow \pi_{1}(\mathcal{X}, \bar{x})$ factors through $\mathbf{G}$ to produce the desired splitting of the above short exact sequence.

If $N \subseteq \pi_{1}(X, x) \rtimes \mathbf{G}$ is as in Theorem 8.3(i), we have

$$
\left(\pi_{1}(X, x) \rtimes \mathbf{G}\right) / N \cong \pi_{1}\left(\mathcal{X}_{\text {mod }}, \bar{x}\right)=\pi_{1}(X / G, \bar{x}) .
$$

We will show that the left hand side is isomorphic to $\pi_{1}(X, x) / K$.

Recall from Lemma 7.5 that, to define $N \subseteq \pi_{1}(\mathcal{X}, \bar{x})$, what we do is that we pick a point $x^{\prime} \in \mathcal{X}$ and identify $\omega_{x^{\prime}}\left(I_{x^{\prime}}\right)$ with various subgroups of $\pi_{1}(\mathcal{X}, \bar{x})$ by taking paths connecting $x^{\prime}$ to $\bar{x}$. For any fixed $x^{\prime} \in \mathcal{X}$, denote the subgroup generated by all these groups by $N_{x^{\prime}} \subset \pi_{1}(\mathcal{X}, \bar{x})$. The group $N$ is then the one generated by all $N_{X^{\prime}}$, $x^{\prime} \in \mathcal{X}$. In fact, to generate $N$ it is enough to take all subgroups of the form $N_{p(y)}$, $y \in X$. Furthermore, to generate $N_{p(y)}$ it suffices to join $p(y)$ to $\bar{x}$ by paths of the from $p(\gamma)$, where $\gamma$ is a path in $X$ joining $y$ to $x$.

The group $N_{p(y)} \subseteq \pi_{1}(\mathcal{X}, x)=\pi_{1}(X, x) \rtimes \mathbf{G}$ can now be explicitly described by

$$
N_{p(y)}=\left\{\left(p\left(\lambda_{g, y, \gamma}\right), g\right) \mid g \in I_{y}, \gamma=\text { path in } X \text { joining } x \text { to } y\right\} .
$$

(This is not completely obvious. The proof requires a little bit of straightforward path chasing that we omit here.) So, to obtain $\left(\pi_{1}(X, x) \rtimes \mathbf{G}\right) / N$ we have to kill all elements of the form $\left(p\left(\lambda_{g, y, \gamma}\right), g\right)$ in $\pi_{1}(X, x) \rtimes \mathbf{G}$. Notice that this includes all elements of the form $(1, g)$, because $\lambda_{g, x \text {, const. }}=1$. Therefore, to obtain $\left(\pi_{1}(X, x) \rtimes \mathbf{G}\right) / N$ we have to kill all elements of the from $(1, g)$ and all elements of the form $\left(p\left(\lambda_{g, y, \gamma}\right), 1\right)$ in $\pi_{1}(X, x) \rtimes \mathbf{G}$. The outcome of this is exactly $\pi_{1}(X, x) / K$. The proof is complete.

Remark 10.2 If we kill all loops of the form $\lambda_{g, x, \gamma}$ in $\pi_{1}(X, x)$ we obtain $\pi_{1}(X, x)_{G}$, the group of coinvariants of the action of $G$ on $\pi_{1}(X, x)$. Therefore, there is a surjective homomorphism $\pi_{1}(X, x)_{G} \rightarrow \pi_{1}(X / G, x)$. This map is not necessarily an isomorphism. For instance, consider the action of $\mathbb{Z} / 2 \mathbb{Z}$ on $S^{1}$ defined by flipping along the $x$-axis. In this case, $\pi_{1}(X, x)_{G}=\mathbb{Z} / 2 \mathbb{Z}$, whereas $\pi_{1}(X / G, x)$ is trivial.

One may wonder if the knowledge of the action of $G$ on $\pi_{1}(X, x)$ is enough to determine $\pi_{1}(X / G, \bar{x})$. The answer is no. For instance, in the above example we 
have $G=\mathbb{Z} / 2 \mathbb{Z}, \pi_{1}(X)=\mathbb{Z}$, the action is $n \mapsto-n$, and we have $\pi_{1}(X / G)=0$. Now, let $X$ be $S^{2}$ with the north and the south poles joined by a straight line. Take $G=\mathbb{Z} / 2 \mathbb{Z}$, and let the action be antipodal. In this case, we have, as in the previous example, $G=\mathbb{Z} / 2 \mathbb{Z}, \pi_{1}(X)=\mathbb{Z}$, and the action of $G$ is $n \mapsto-n$. However, $X / G$ is homotopy equivalent to $\mathbb{R} P^{2}$, so $\pi_{1}(X / G)=\mathbb{Z} / 2 \mathbb{Z}$.

Indeed, it is true in general that, if $G$ acts freely away from $x$, then $\pi_{1}(X, x)_{G} \rightarrow$ $\pi_{1}(X / G, x)$ is an isomorphism. This follows easily from Theorem 10.1. The rest of this section is devoted to understanding the map $\pi_{1}(X, x)_{G} \rightarrow \pi_{1}(X / G, x)$ in general.

\subsection{Another formulation of the explicit formula}

The subgroup $K$ appearing in Theorem 10.1 may look a bit too complicated to compute in general, because it seems to require a detailed knowledge of the various fixed point sets $X^{g}$. In the next theorem, we build on the idea discussed in Remark 10.2 and give a more efficient formula for $\pi_{1}(X / G)$.

Construction For every $g \in G$, choose a set of paths $\left\{\gamma_{g, i}\right\}_{i \in \pi_{0} X^{g}}$ with the property that $\gamma_{g, i}$ starts from $x$ and ends on the path component of $X^{g}$ corresponding to $i$. Let $\pi_{1}(X, x)_{G}$ be the group of coinvariants of the action of $G$ on $\pi_{1}(X, x)$, that is, the largest quotient of $\pi_{1}(X, x)$ on which $G$ acts trivially. Denote the image of $\gamma_{g, i}\left(g \gamma_{g, i}\right)^{-1}$ in $\pi_{1}(X, x)_{G}$ by $\bar{\lambda}_{g, i}$. Let $\bar{K} \subseteq \pi_{1}(X, x)_{G}$ be the normal subgroup generated by $\left\{\bar{\lambda}_{g, i}\right\}_{g \in G, i \in \pi_{0} X^{g}}$ (that is, the smallest normal subgroup of $\pi_{1}(X, x)_{G}$ that contains all $\bar{\lambda}_{g, i}$ ).

Remark 10.3 The generating loops we used to define the group $\bar{K}$ are superfluous. Indeed, we could define $\bar{K}$ to be the normal subgroup generated by $\left\{\bar{\lambda}_{g, i}\right\}$, where $g$ runs in $G$ and $i$ runs in $\pi_{0} X^{g} / C(g)$. Here, $C(g)$ stands for the centralizer of $g$ in $G$.

Theorem 10.4 Let $X$ and $G$ be as in Theorem 9.1 (also see Remark 9.2). Assume that there is $x \in X$ which is fixed by the action. Let $\bar{K}$ be the subgroup of $\pi_{1}(X, x)_{G}$ defined above. Then we have a natural isomorphism

$$
\pi_{1}(X, x)_{G} / \bar{K} \stackrel{\sim}{\longrightarrow} \pi_{1}(X / G, \bar{x}) .
$$

Proof We will show that $\bar{K}$ is the image of $K$ in $\pi_{1}(X, x)_{G}$, where $K$ is as in Theorem 10.1. The first observation is that $\lambda_{g, y, \gamma}=\lambda_{g, y^{\prime}, \gamma^{\prime}}$, if $\gamma$ and $\gamma^{\prime}$ are homotopic via a homotopy that is relative to both $\{x\}$ and $X^{g}$. Therefore, to define $K$ it is enough to choose a set of representative elements $\left\{y_{g, i}\right\}_{i \in \pi_{0} X^{g}}$ for path components of $X^{g}$ and only use paths $\lambda_{g, \gamma, y}$ whose end points $y$ belong to this representative set. 
We now fix $g$ and $y$ and analyze what happens if we replace the path $\gamma$ with another path $\gamma^{\prime}$ joining $x$ to $y$. Set $\alpha:=\gamma^{\prime} \gamma^{-1} \in \pi_{1}(X, x)$ and $\beta:=\alpha(g \alpha)^{-1}$. It is easy to check that we have the equality

$$
\lambda_{g, y, \gamma^{\prime}}=\alpha \lambda_{g, y, \gamma} \alpha^{-1} \beta
$$

in $\pi_{1}(X, x)$. Since the image of $\beta$ in $\pi_{1}(X, x)_{G}$ is trivial, the images $\bar{\lambda}_{g, y, \gamma}$ and $\bar{\lambda}_{g, y, \gamma^{\prime}}$ of these loops are conjugate in $\pi_{1}(X, x)_{G}$. Thus, a normal subgroup that contains one will necessarily contain the other. Therefore, as far as generating a normal subgroup is concerned, we can choose either of $\bar{\lambda}_{g, y, \gamma}$ and $\bar{\lambda}_{g, y, \gamma^{\prime}}$. That is, the normal subgroup of $\pi_{1}(X, x)_{G}$ generated by the elements $\bar{\lambda}_{g, i}$ used in the construction of $\bar{K}$ is equal to the normal subgroup generated by all $\bar{\lambda}_{g, y, \gamma}$. In other words, $\bar{K}$ is exactly the image of $K$ in $\pi_{1}(X, x)_{G}$.

Corollary 10.5 Let $X$ and $G$ be as in Theorem 9.1 (also see Remark 9.2). Assume that there is $x \in X$ which is fixed by the action. Also, assume that for every $g \in G$, the fixed set $X^{g}$ is path-connected. (It is enough to assume that $X^{g} / C(g)$ is pathconnected.) Then we have an isomorphism

$$
\pi_{1}(X, x)_{G} \stackrel{\sim}{\longrightarrow} \pi_{1}(X / G, \bar{x}) .
$$

Proof Note that the fixed point set $X^{g}$ contains $x$, for every $g \in G$. Hence, we can choose the paths $\left\{\gamma_{g, i}\right\}$ to be the constant paths (see the construction of $\bar{K}$ just before Theorem 10.4 for notation). This way, the elements $\bar{\lambda}_{g, i}$ will be trivial. Therefore, the group $\bar{K}$ is trivial.

Example 10.6 Let $G$ be a compact Lie group, and consider the conjugation action of $G$ on its classifying space $B G$. (Here, $B G$ stands for the classical classifying space, not the stack one.) Using the standard model for $B G$, namely, the geometric realization of the simplicial space associated to $G$, we see that, for every $g \in G$, the fixed set of $g$ is homeomorphic to $B(C(g))$, where $C(g)$ is the centralizer of $g$. It follows that the fixed point sets are all connected. So, by Corollary 10.5, we have

$$
\pi_{1}(B G / G) \cong\left(\pi_{1} B G\right)_{G} \cong\left(G / G^{0}\right)^{\mathrm{ab}}=\pi_{0}(G)^{\mathrm{ab}} .
$$

Remark 10.7 Theorem 10.1 and Theorem 10.4 can be generalized to the case where the base point $x$ is not necessarily fixed by the entire action. For instance, we can take $Y=\operatorname{Cone}(G \cdot x) \cup_{G \cdot x} X$ with its induced $G$ action. The tip of the cone $\operatorname{Cone}(G \cdot x)$ is now fixed by the entire action. Hence, Theorems 10.1 and 10.4 apply. Note that $Y / G \cong[0,1] \cup_{\{0\}} X / G$ is homotopy equivalent to $X / G$. 


\section{Relation with Armstrong's work}

In [2] Armstrong gives a formula for the fundamental group of the coarse quotient $X / G$ of a group $G$ acting faithfully on a topological space $X$. To do so, he makes three assumptions on the group action. We will not go over Armstrong's result. Instead, we translate his conditions $\mathbf{A}, \mathbf{B}$ and $\mathbf{C}$ into stack language and deduce the stack version of his result, generalizing the main theorem of [2].

In what follows, $\mathcal{X}$ is a connected locally path-connected topological stack.

(A) The moduli map $\pi: \mathcal{X} \rightarrow \mathcal{X}_{\text {mod }}$ has the homotopy path lifting property. That is, for any point $x \in \mathcal{X}$ and any path $\gamma$ initiating from its image $\bar{x} \in \mathcal{X}_{\text {mod }}$, there is a path $\tilde{\gamma}$ initiating at $x$ such that $\pi(\tilde{\gamma})$ is homotopic to $\gamma$ rel. $\bar{x}$.

(C) Let $N \subseteq \pi_{1}(\mathcal{X}, x)$ be as in Lemma 7.5. There is a covering stack of $\mathcal{X}$ corresponding to $N$, and this covering stack is free.

Proposition 11.1 Assume that (A) and (C) hold. Then we have a natural isomorphism

$$
\pi_{1}(\mathcal{X}, x) / N \stackrel{\sim}{\longrightarrow} \pi_{1}\left(\mathcal{X}_{\text {mod }}, x\right) .
$$

Proof First of all, note that, by $(\mathbf{C})$, the prodiscrete topology Free on $\pi_{1}(\mathcal{X}, x)$ is the same as the topology generated by $N$. So $\pi_{1}^{\prime}(\mathcal{X}, x)_{\text {Free }}^{\wedge}=\pi_{1}(\mathcal{X}, x) / N$. Let $f:(\mathcal{Y}, y) \rightarrow(\mathcal{X}, x)$ be the covering stack corresponding to $N$. By $(\mathbf{C}), \mathcal{Y}_{\text {mod }} \rightarrow \mathcal{X}_{\text {mod }}$ is a covering map. Condition (A) implies that $\pi: \mathcal{Y} \rightarrow \mathcal{Y}_{\text {mod }}$ also has the homotopy path lifting property. Therefore, $\pi_{1}(\mathcal{Y}, y) \rightarrow \pi_{1}\left(\mathcal{Y}_{\text {mod }}, y\right)$ is surjective.

On the other hand, it follows from the commutative diagram

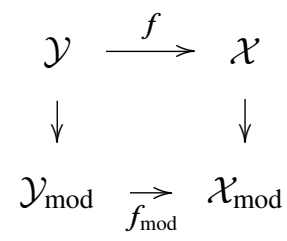

that the image of $\pi_{1}(\mathcal{Y}, y)$ in $\pi_{1}\left(\mathcal{Y}_{\bmod }, y\right)$ is trivial (note that $f_{\text {mod }}$ is a covering map). This means that $\pi_{1}\left(\mathcal{Y}_{\text {mod }}, y\right)$ is trivial, so $\mathcal{X}_{\text {mod }}$ has a universal cover. Therefore, the full topology on $\pi_{1}\left(\mathcal{X}_{\text {mod }}, x\right)$ is discrete, ie, $\pi_{1}^{\prime}(\mathcal{X}, x)=\pi_{1}(\mathcal{X}, x)$. On the other hand, by definition, $N$ is open in the full topology of $\pi_{1}(\mathcal{X}, x)$. The result now follows from Proposition 8.1(ii).

In practice, conditions (A) and (C) may fail for pathological reasons. What condition B of Armstrong requires is the existence of another topological stack $\mathcal{X}^{\prime}$ together 
with a map $\mathcal{X} \rightarrow \mathcal{X}^{\prime}$ such that $\mathcal{X}^{\prime}$ satisfies (A) and (C) and that the induced map

$\mathcal{X}_{\text {mod }} \rightarrow \mathcal{X}_{\text {mod }}^{\prime}$ is so that every fiber has trivial topology. Since $\mathcal{X}_{\text {mod }} \rightarrow \mathcal{X}_{\text {mod }}^{\prime}$ is a homotopy equivalence, to compute $\pi_{1}(\mathcal{X})$ it is enough to compute $\pi_{1}\left(\mathcal{X}^{\prime}\right)$, and for this we can apply the above proposition.

A typical example of the above situation is the following. Assume that $G$ is a group acting on a space $X$, and let $\mathcal{X}=[X / G]$. It may happen that the group $G$ is not nice enough so as to be able to apply Theorems 9.1 or 9.6. However, if we can realize $G$ as a dense subgroup of a Lie group $G^{\prime}$ and extend the action to $G^{\prime}$, then chances are that $\mathcal{X}^{\prime}=\left[X / G^{\prime}\right]$ is a better behaved stack to which the discussion of the previous paragraph applies.

Example 11.2 Let $\mathbb{Q}$ (viewed as a discrete group) act on $\mathbb{R}$ by, translation and let $\mathcal{X}=[\mathbb{R} / \mathbb{Q}]$. Then $\mathcal{X}^{\prime}=[\mathbb{R} / \mathbb{R}]$ together with the obvious map $\mathcal{X} \rightarrow \mathcal{X}^{\prime}$ has the above property.

What happens in this example is that we can replace $\mathbb{Q}$ by its closure in the group $\operatorname{Iso}(\mathbb{R}, \mathbb{R})$, thereby turning an awkward action of an infinite discrete group into a nice action of a connected Lie group.

In general, when $G$ is a subgroup of the group of isometries of a locally compact metric space $X$, then the action of the closure $\bar{G}$ (in the compact-open topology) satisfies the above property [2, Corollary 2]. That is, the fibers of the map $q: X / G \rightarrow X / \bar{G}$ have trivial topology (hence $q$ is a homotopy equivalence). In this situation, Armstrong's trick comes handy.

\section{References}

[1] MA Armstrong, The fundamental group of the orbit space of a discontinuous group, Proc. Cambridge Philos. Soc. 64 (1968) 299-301 MR0221488

[2] M A Armstrong, Calculating the fundamental group of an orbit space, Proc. Amer. Math. Soc. 84 (1982) 267-271 MR637181

[3] H Bass, Covering theory for graphs of groups, J. Pure Appl. Algebra 89 (1993) 3-47 MR1239551

[4] G E Bredon, Introduction to compact transformation groups, Pure and Applied Math. 46, Academic Press, New York (1972) MR0413144

[5] R Brown, Topology. A geometric account of general topology, homotopy types and the fundamental groupoid, second edition, Ellis Horwood Series: Math. and its Applications, Ellis Horwood Ltd., Chichester (1988) MR984598

Algebraic $8 \mathcal{G}$ Geometric Topology, Volume 8 (2008) 
[6] A Grothendieck, editor, M Raynaud, Revêtements étales et groupe fondamental, Lecture Notes in Math. 224, Springer, Berlin (1971) MR0354651Séminaire de Géométrie Algébrique du Bois Marie 1960-1961 (SGA 1)

[7] P J Higgins, J Taylor, The fundamental groupoid and the homotopy crossed complex of an orbit space, from: "Category theory (Gummersbach, 1981)", Lecture Notes in Math. 962, Springer, Berlin (1982) 115-122 MR682948

[8] J-i Nagata, Modern general topology, second edition, North-Holland Math. Library 33, North-Holland Publishing Co., Amsterdam (1985) MR831659

[9] B Noohi, Foundations of topological stacks I arXiv:math/0503247v1

[10] R S Palais, On the existence of slices for actions of non-compact Lie groups, Ann. of Math. (2) 73 (1961) 295-323 MR0126506

[11] F Rhodes, On the fundamental group of a transformation group, Proc. London Math. Soc. (3) 16 (1966) 635-650 MR0203715

[12] L Ribes, P Zalesskii, Profinite groups, Ergebnisse der Math. und ihrer Grenzgebiete. 3. Folge. A Series of Modern Surveys in Math. [Results in Math. and Related Areas. 3rd Series.] 40, Springer, Berlin (2000) MR1775104

[13] A Weinstein, Linearization of regular proper groupoids, J. Inst. Math. Jussieu 1 (2002) 493-511 MR1956059

[14] N T Zung, Proper groupoids and momentum maps: linearization, affinity, and convexity, Ann. Sci. École Norm. Sup. (4) 39 (2006) 841-869 MR2292634

Florida State University, Department of Mathematics

Tallahassee, Florida 32306-4510, USA

behrang@alum.mit.edu

http://www.math.fsu.edu/ noohi/

Received: 11 January 2008 Revised: 14 June 2008 